\title{
A Refined Zigzag Beam Theory for Composite and Sandwich Beams
}

\author{
Alexander Tessler \\ Structural Mechanics and Concepts Branch - NASA Langley Research Center \\ Mail Stop 190, Hampton, Virginia, 23681 - 2199, U.S.A. \\ Marco Di Sciuva, Marco Gherlone ${ }^{1}$ \\ Department of Aeronautics and Space Engineering - Politecnico di Torino \\ Corso Duca degli Abruzzi 24 \\ 10129, Torino, Italy
}

\begin{abstract}
A new refined theory for laminated composite and sandwich beams that contains the kinematics of the Timoshenko Beam Theory as a proper baseline subset is presented. This variationally consistent theory is derived from the virtual work principle and employs a novel piecewise linear zigzag function that provides a more realistic representation of the deformation states of transverse-shear flexible beams than other similar theories. This new zigzag function is unique in that it vanishes at the top and bottom bounding surfaces of a beam. The formulation does not enforce continuity of the transverse shear stress across the beam's cross-section, yet is robust. Two major shortcomings that are inherent in the previous zigzag theories, shear-force inconsistency and difficulties in simulating clamped boundary conditions, and that have greatly limited the utility of these previous theories are discussed in detail. An approach that has successfully resolved these shortcomings is presented herein. Exact solutions for simply supported and cantilevered beams subjected to static loads are derived and the improved modelling capability of the new "zigzag” beam theory is demonstrated. In
\end{abstract}


particular, extensive results for thick beams with highly heterogeneous material lay-ups are discussed and compared with corresponding results obtained from elasticity solutions, two other "zigzag" theories, and high-fidelity finite element analyses. Comparisons with the baseline Timoshenko Beam Theory are also presented. The comparisons clearly show the improved accuracy of the new, refined "zigzag” theory presented herein over similar existing theories. This new theory can be readily extended to plate and shell structures, and should be useful for obtaining relatively low-cost, accurate estimates of structural response needed to design an important class of high-performance aerospace structures.

\section{KEYWORDS}

Timoshenko beam theory, Zigzag kinematics, Shear deformation, Composite beams, Sandwich beams, Virtual work principle

1 Author to whom correspondence should be sent: Tel. +39-0115646817, FAX +39-0115646899, EMAIL marco.gherlone@polito.it 


\section{NOMENCLATURE}

A, $2 h, L$

$2 h^{(k)}$

$q, F$

$\left(T_{x r}, T_{z r}\right)$

$\left(u_{x}^{(k)}, \quad u_{z}^{(k)}\right)$

$(u, \theta, \psi, w)$

$\phi^{(k)}$

$\left(\varepsilon_{x}^{(k)}, \gamma_{x z}^{(k)}\right)$

$\left(\sigma_{x}^{(k)}, \tau_{x z}^{(k)}\right)$

$\left(E_{x}^{(k)}, G_{x z}^{(k)}\right)$

$\lambda_{A}, \lambda_{0}$

$\xi^{(k)}$

$u^{(k)}$

$\left(N_{x}, \quad M_{x}, \quad M_{\phi}, \quad V_{x}, V_{\phi}\right)$

$A_{11}, B_{i j}, D_{i j}$, and $Q_{i j}^{\lambda}$
$=$ beam's cross-sectional area, depth, and span

$=$ thickness of the $k$-th layer

$=$ transverse pressure loading [force/length] and tip shear force

$=$ axial and shear tractions prescribed at the ends of beam

$$
x_{r}(r=a, b)
$$

$=$ axial and transverse components of the displacement vector in the $k$-th layer

$=$ kinematic variables of zigzag theory

$=$ zigzag function

$=$ axial and transverse shear strain in the $k$-th layer

$=$ axial and transverse shear stress in the $k$-th layer

$=$ axial and shear modulus of the $k$-th layer

$=$ penalty factors

$=$ local $(k$-th layer $)$ thickness coordinate

$=$ normalized axial displacement along the interface between the $k$-th and $(k+1)$ layers

$=$ stress resultants of the refined zigzag beam theory

$=$ constitutive stiffness coefficients of the refined zigzag beam theory 


\section{INTRODUCTION}

Performance and weight advantages of advanced composite materials have led to their sustained and increased application to military and civilian aircraft, aerospace vehicles, naval and civil structures. To design efficient and reliable composite structures, improved analytical and computational methods that accurately incorporate principal non-classical effects are necessary. In relatively thick and/or heterogeneous beams, shear deformation may influence, to a significant degree, such design-significant response quantities as the normal stresses, deflection, vibration modes, and natural frequencies. The inherent assumptions of classical deformation theories generally render such theories less than adequate for application to advanced composites. This shortcoming is particularly manifested in relatively thick structures with material layers that exhibit large differences in the transverse shear properties, often leading to non-conservative predictions for deformation, stresses, and natural frequencies. It is further noted that, in these classical shear deformation models, transverse shear stresses fail to satisfy equilibrium conditions at the layer interfaces.

The two key assumptions of Bernoulli-Euler beam (known as the Kirchhoff-Love hypotheses in plates and shells) are those of zero transverse shear strain and non-deformable transverse normal - the assumptions that are fully consistent for the bending of very slender beams that exhibit negligibly small shear deformations. The bending deformation may thus be defined in terms of a single deflection variable. Here Hooke’s law only leads to a zero transverse shear stress. Instead, a beam equilibrium equation is used to obtain the shear force from which an average shear stress is computed. Timoshenko ${ }^{1}$ introduced an additional kinematic variable, the bending rotation, to account for shear deformation in an average sense while retaining the non-deformable normal assumption. This improvement over the classical beam theory allows the transverse shear stress to be obtained from Hooke's law, and extends the range of applicability to thick beams.

Timoshenko beam theory, and analogous shear-deformation theories for plate and shell structures, 
has been widely used in structural analysis of homogeneous and composite beam-type structures. The theory produces inadequate predictions, however, when applied to relatively thick composite laminates composed of material layers that have highly dissimilar stiffness characteristics. Even with a judiciously chosen shear correction factor, which is dependent on the stacking sequence, Timoshenko theory tends to underestimate, often substantially, the axial stress on the top and bottom surfaces. Moreover, along layer interfaces, the transverse shear stress often exhibits excessively erroneous discontinuities. The reason for these difficulties might be traced to a higher complexity of the 'true' displacement field across a highly heterogeneous cross-section. Clearly, the linear throughthickness displacement assumption for the axial displacement is the main shortcoming of Timoshenko theory when the modelling of complex material systems is undertaken.

Higher-order terms, with respect to the thickness coordinate, have been added to the in-plane displacements and, in some cases, to the transverse displacement. This leads to the so-called higherorder theories that are also commonly known as equivalent single-layer theories ${ }^{2}$. While notable response improvements have been achieved with several of such theories, they generally fall short as far as predicting correct shear and axial stress behaviour in highly heterogeneous lay-ups in moderately thick laminates and high-frequency dynamics.

Departing from the equivalent single-layer modelling assumptions, layer-wise theories assume that the behaviour of a laminate is due to an assembly of the individual layers whose kinematic fields are independently described while satisfying certain physical continuity constraints ${ }^{2}$. The increased kinematic freedom provided by the layer-wise schemes enable the enforcement of the interlaminar stress continuity conditions and the modelling of the zigzag displacement through a laminate thickness. The major drawback of such theories, however, is that the number of kinematic variables is dependent on the number of layers; thus, for thick laminates with a large number of plies, a great number of variables results, making such approaches computationally unattractive. Notable early contributions to layer-wise schemes are those due to Ambartsumian ${ }^{3}$ and Sun and Whitney ${ }^{4}$. While providing relatively accurate approximations, these theories possess a large number of variables and 
are particularly cumbersome to implement within a displacement-based finite element method ${ }^{5}$.

The so-called zigzag theories constitute a special sub-class of layer-wise theories. They assume a zigzag pattern for the in-plane displacements and enforce the continuity of the shear stresses across the entire laminate thickness. They give rise to bending theories based on the same number of kinematic variables regardless of the number of layers in a laminate. Thus, the early efforts of Di Sciuva $^{6-8}$ and Murakami ${ }^{9}$ employed zigzag-like displacement fields that satisfy a priori the transverse shear stress and displacement continuity conditions at the layer interfaces while keeping the number of kinematic variables independent of the number of layers. Di Sciuva ${ }^{10-11}$ also demonstrated that such models are well-suited for finite element approximations.

In Di Sciuva's earlier efforts ${ }^{6-7}$, a form of shear deformation theory is augmented by adding a piecewise linear ("zigzag”) function to the in-plane displacement. To retain only the kinematic variables of the classical theory, a constant shear stress is enforced across the entire laminate thickness. This procedure led to the desired enhancement in the axial displacement and simultaneously achieved the shear stress continuity along layer interfaces. Furthermore, for homogeneous cross-sections the zigzag shape function vanishes identically, thus resorting back to a shear deformation theory. Di Sciuva ${ }^{12-13}$ also introduced further enhancements to the zigzag model by adding to a zigzag function a cubic in-plane displacement. The Di Sciuva theories require $C^{1}-$ continuous shape functions for formulating suitable finite elements. Such approximation schemes are significantly less attractive, especially for plate and shell finite elements, than the $C^{0}$-continuous displacement interpolations associated with Timoshenko-type theories.

Exploring the new linear ${ }^{14}$ and cubic $^{15}$ zigzag beam models with a view on $C^{0}$-continuous finite elements, Averill modified the Di Sciuva approach by starting with Timoshenko theory, adding an additional kinematic variable associated with a zigzag function, and by introducing an ad hoc penalty term in the variational principle. The penalty term serves to enforce the continuity of transverse shear stress across the cross-section in a limiting sense.

Di Sciuva's theory runs into theoretical difficulties in an attempt to interpret the physical 
significance of the shear stress associated with the theory. The difficulty is especially evident at the clamped support, where the cross-sectional area integral of the shear stress, obtained from constitutive relations, does not correspond to the total shear force. Thus, the correct shear force and the average shear stress can be determined from an equilibrium equation relating the shear force to the derivative of the bending moment, as in Bernoulli-Euler theory. Averill's theory also suffers from its inability to model correctly a clamped boundary condition, where it predicts erroneously that the transverse shear stress and the corresponding resultant force vanish. To alleviate this anomaly, Averill proposed a boundary condition compromise at the expense of variational consistency of the theory, in which a kinematic variable representing the amplitude of the zigzag displacement is left out of the variationally required boundary condition. Consequently, extensive analytic and numerical studies that have been conducted primarily focused on beams and plates with simply supported boundaries ${ }^{6-}$ ${ }^{7,12-15}$. Recently, a zigzag plate analysis was discussed for clamped plates ${ }^{16}$; however, no results were presented for the shear stresses along the clamped edges.

Scrutiny of the zigzag theories discussed herein has revealed some serious shortcomings. The aim of the present study is to present a new refined zigzag theory that is free of these shortcomings and amenable to finite element implementation. In particular, the present paper discusses a new refined zigzag beam theory of Tessler, Di Sciuva and Gherlone ${ }^{17,18}$ that is consistently derived from the virtual work principle, by refining the ideas of Timoshenko, Di Sciuva, and Averill. The key attributes of the present theory are, first, the proposed zigzag function vanishes at the top and bottom surfaces of the beam and does not require full shear-stress continuity across the laminated-beam depth. Second, all boundary conditions, including the fully clamped condition, can be modelled adequately. And third, the theory requires only $C^{0}$-continuous kinematics for finite element modelling, as do elements based on the theories of Timoshenko ${ }^{1}$, Mindlin ${ }^{19}$, and Reissner ${ }^{20}$. This latter attribute lends itself to developing computationally efficient and robust beam, plate, and shell finite elements. Overall, the theory appears as a natural extension of Timoshenko theory to laminated composite beams, and it is devoid of the drawbacks of the zigzag theories discussed previously. 
In the remainder of the paper, the concept of zigzag kinematic assumptions is first described. Then the original zigzag schemes of Di Sciuva and Averill are elaborated in detail, and their deficiencies with respect to the transverse shear properties and clamped boundary conditions are highlighted. A unique zigzag function is then introduced to formulate the basis for the refined zigzag theory, giving rise to the transverse shear stress that has a piecewise constant distribution across the laminate thickness. As an added explanation of the underlying reasons for the drawbacks of Averill's formulation, a penalized form of the constitutive equations is introduced within the present theory. The equations of equilibrium and associated boundary conditions are then derived from the virtual work principle. Finally, the refined zigzag theory is assessed quantitatively by way of exact solutions for simply supported and cantilevered composite and sandwich beams. Thick beams composed of highly heterogeneous material lay-ups are considered. Comparisons are made with several beam theories, exact elasticity solutions, and results obtained with high-fidelity, two-dimensional elasticity finite element models.

This paper is an enhanced version of the article ${ }^{18}$ presented at the VI International Symposium on Advanced Composites and Applications for the New Millennium, held in Corfù, Greece, in May 2007.

\section{CONCEPT OF ZIGZAG KINEMATICS}

The response of heterogeneous, anisotropic laminated beams exhibiting the bending, shear and axial deformations is generally manifested by a zigzag-like through-thickness displacement field. Here the axial displacements are dominant, mainly in thick and/or heterogeneous beams, in their influence on the bending strain and stress. The cross-section of the deformed beam tends to distort according to a piecewise $C^{0}$-continuous pattern, having discontinuous thickness-direction derivatives along the material layer interfaces. Within the individual material layers, the displacement distributions are generally nonlinear and sufficiently smooth. Such observations, based on exact 
elasticity solutions (e.g., Pagano ${ }^{21}$ ), prompted Di Sciuva ${ }^{7}$ to add a zigzag kinematic term to a firstorder shear deformation theory in which shearing angles appear as independent variables. Following Di Sciuva’s work, Averill ${ }^{14}$ proposed a similar zigzag enhancement for application to beam bending analysis of composite laminates using the standard form of Timoshenko theory in which the bending rotation is represented by an independent variable appearing in the axial displacement expansion. In what follows, the essential aspects of Di Sciuva and Averill zigzag models are examined in order to set the stage for the new refined zigzag bending theory. The theoretical anomalies encountered by these earlier zigzag models are discussed in sufficient detail.

Consider a narrow beam with the cross sectional area $A$. The beam is made of $N$ orthotropic material layers that are perfectly bonded to each other and are parallel to the $\mathrm{x}$ axis. For the sake of the present discussion, only planar deformations are considered under the static loading which includes a distributed transverse pressure $q(x)$ (units of force/length) and the prescribed axial $\left(T_{x a}, T_{x b}\right)$ and shear $\left(T_{z a}, T_{z b}\right)$ tractions at the two reference cross sections $x=x_{a}$ and $x_{b}$ (refer to Figure 1 ).

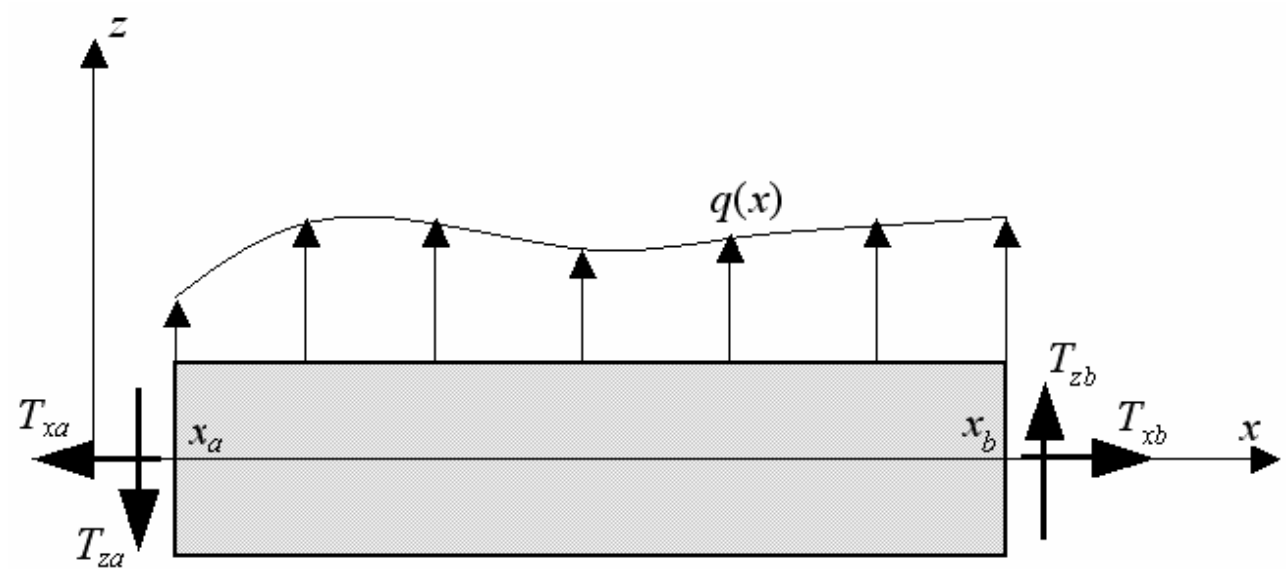

Figure 1. Beam subjected to transverse loading and end tractions.

For any material point within the $k$-th layer, the displacement vector, which is general enough to describe the kinematics of the Di Sciuva, Averill, and the present refined zigzag theory, is expressed herein as 


$$
\begin{aligned}
& u_{x}^{(k)}(x, z)=u(x)+z \theta(x)+\phi^{(k)}(z) \psi(x) \\
& u_{z}^{(k)}(x, z)=w(x)
\end{aligned}
$$

In Equation (1), $z \in[-h, h]$ is a thickness coordinate defining the position of the reference $\mathrm{x}$-axis half way through the laminate thickness with $2 h$ denoting the total beam thickness, $u(x)$ is the uniform axial displacement, $\theta(x)$ is the bending rotation, $w(x)$ is the deflection, $\psi(x)$ is the amplitude of the zigzag contribution to the axial displacement, and $\phi^{(k)}(z)$ denotes a piecewise linear zigzag function yet to be established. When dynamic effects are considered, the four kinematic variables are also functions of time. If either $\phi^{(k)}(z)=0$ or $\psi(x)=0$, the kinematic assumptions of Equation (1) correspond to Timoshenko theory if $\theta(x)$ is an independent variable. Note that depending on the specific theory used, the four kinematic variables may have slightly different physical interpretations. In Figure 2, notation for a three-layered beam is shown together with a general distribution of the zigzag function, $\phi^{(k)}$. More generally, if delaminations along the layer interfaces are modeled, the zigzag function may be represented having jump conditions along such interfaces ${ }^{22}$.

The linear strain-displacement relations give rise to the strain expressions

$$
\begin{aligned}
& \varepsilon_{x}^{(k)}(x, z) \equiv u_{x, x}^{(k)}=u_{, x}+z \theta_{, x}+\phi^{(k)} \psi_{, x} \\
& \gamma_{x z}^{(k)}(x, z) \equiv u_{x, z}^{(k)}+u_{z, x}^{(k)}=\gamma+\beta^{(k)} \psi
\end{aligned}
$$

where $\beta^{(k)} \equiv \phi_{, z}^{(k)}$, and $\gamma \equiv w_{, x}+\theta$ represents an average shear strain (or shearing angle) within the assumptions of Timoshenko theory. Note that, since $\phi^{(k)}(z)$ is piecewise linear, $\beta^{(k)}$ is a piecewise constant function, i.e., it is constant across each material layer. 
Assuming the principal material axes are coincident with the Cartesian coordinates, Hooke's stressstrain relations for the $k$-th orthotropic layer have the standard form

$$
\left\{\begin{array}{l}
\sigma_{x}^{(k)} \\
\tau_{x z}^{(k)}
\end{array}\right\}=\left[\begin{array}{cc}
E_{x}^{(k)} & 0 \\
0 & G_{x z}^{(k)}
\end{array}\right]\left\{\begin{array}{l}
\varepsilon_{x}^{(k)} \\
\gamma_{x z}^{(k)}
\end{array}\right\}
$$

where $E_{x}^{(k)}$ and $G_{x z}^{(k)}$ denote the axial and shear moduli of the $k$-th layer.

\subsection{Transverse Shear Stress}

To facilitate further discussions, it is now convenient to define the difference function $\eta(x)$ as

$$
\eta \equiv \gamma-\psi
$$

This leads to the expressions for the transverse shear strain and stress in terms of the $\gamma(x)$ and $\eta(x)$ functions

$$
\begin{aligned}
& \gamma_{x z}^{(k)}=\left(1+\beta^{(k)}\right) \gamma-\beta^{(k)} \eta \\
& \tau_{x z}^{(k)}=G_{x z}^{(k)}\left(1+\beta^{(k)}\right) \gamma-G_{x z}^{(k)} \beta^{(k)} \eta
\end{aligned}
$$

\subsection{Di Sciuva’s Zigzag Model}

Di Sciuva's zigzag model ${ }^{7}$, originally developed in the context of plate bending, can be specialized for the beam kinematics, Equation (1), by way of the following variable substitutions

$$
\theta=\psi-w_{, x}
$$


and where the $z$ coordinate is replaced with $(z+h)$ in order to adhere to the precise kinematic definitions in ${ }^{7}$. Thus, the axial displacement of Di Sciuva's model has the form

$$
u_{x}^{(k)}(x, z)=u(x)+(z+h)\left[\psi(x)-w_{, x}(x)\right]+\phi_{D S}^{(k)}(z) \psi(x)
$$

where the transverse displacement is the same as in Equation (1), and where $\phi_{D S}^{(k)}$ now designates the specific zigzag function used within this model. The above kinematics give rise to the shear strain and stress that are piecewise constant (i.e., they are constant within the individual plies) and which are defined exclusively in terms of the amplitude function $\psi$

$$
\begin{aligned}
& \gamma_{x z}^{(k)}=\left(1+\beta_{D S}^{(k)}\right) \psi \\
& \tau_{x z}^{(k)}=G_{x z}^{(k)}\left(1+\beta_{D S}^{(k)}\right) \psi
\end{aligned}
$$

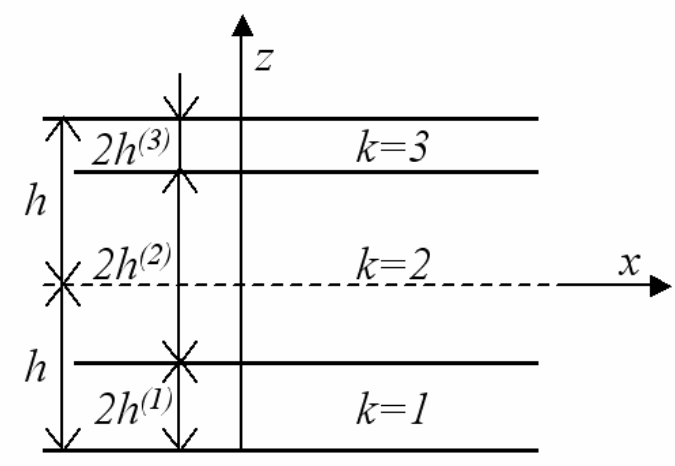

(a)

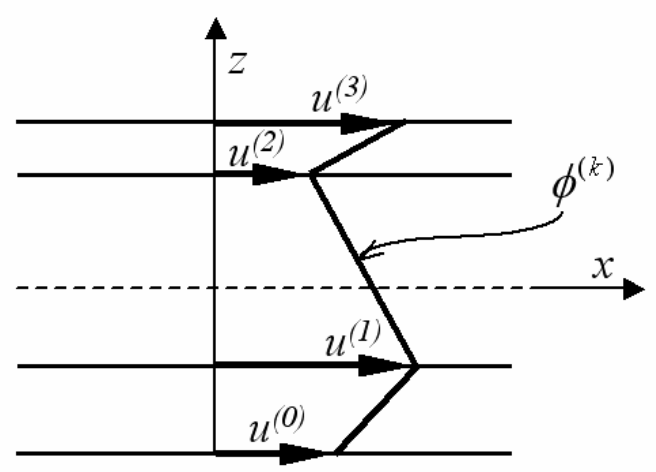

(b)

Figure 2. (a) Layer notation for a three-layered laminate, and (b) a corresponding generic zigzag function defined in terms of interfacial axial displacements, $u^{(i)}(i=0,1, \ldots, N)$.

To determine the $\phi_{D S}^{(k)}$ function, Di Sciuva employs a set of explicit stress-continuity constraints along the layer interfaces, insisting that all layers have the same (constant) transverse shear stress 


$$
\tau_{x z}^{(k)}=\tau_{x z}^{(k+1)}, \quad k=1, \ldots, N-1
$$

Since the above equations impose only $N-1$ constraints, and there are $N+1$ interfacial displacements $u^{(i)}(i=0,1, \ldots, N)$ which define $\phi_{D S}^{(k)}$, the zigzag function is set to vanish across the entire bottom layer ( $k=1$, refer to Figure 3(a)). More generally, it is straightforward to select any layer in which a zigzag function may vanish ${ }^{22}$. Henceforth, determining a zigzag function by way of zeroing out (or fixing) a single layer contribution will be referred to as a fixed-layer zigzag function method.

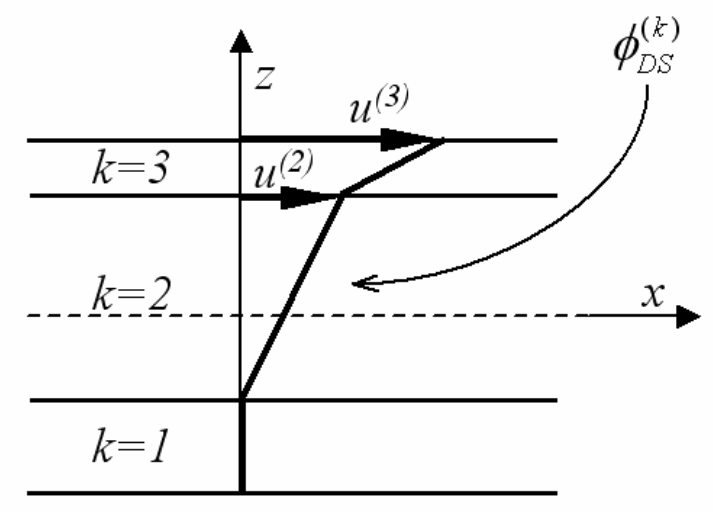

(a)

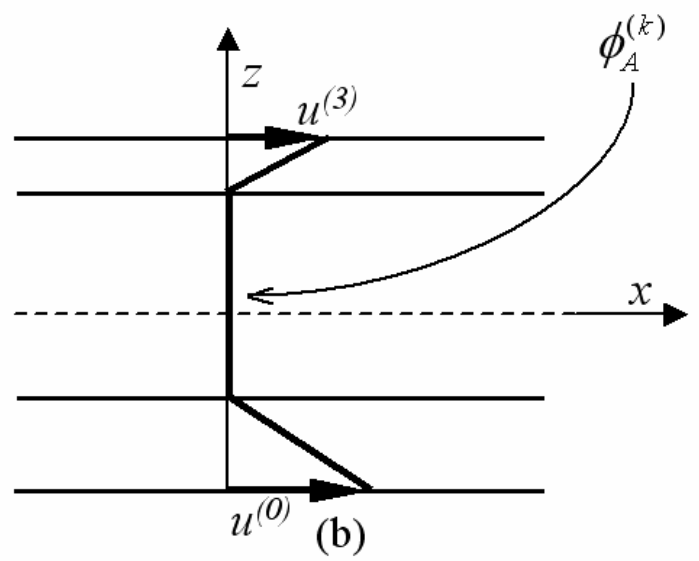

Figure 3. (a) Di Sciuva ${ }^{7}$ and (b) Averill ${ }^{14}$ zigzag functions.

The resulting transverse shear stress is uniform through the thickness. If the first layer $(k=1)$ is fixed in the definition of the zigzag function $\left(\phi_{D S}^{(1)}=\beta_{D S}^{(1)}=0\right)$, as depicted in Figure 3(a), then from Equation (9), the definition of $\psi$ as the shear strain in the first layer becomes evident

$$
\psi=\gamma_{x z}^{(1)}
$$

and, taking into account Equation (11), the shear stress in all layers simply equals the stress in the first 
layer

$$
\tau_{x z}^{(k)}=G_{x z}^{(1)} \gamma_{x z}^{(1)} \quad(k=1, \ldots, N)
$$

As seen from Equation (12), all shear stresses in this model depend on the shear modulus and shear strain in the first layer; hence the validity of this result is questionable. More generally, the shear stress continuity enforcement, Equation (10), leads to the lack of invariance with respect to the choice of the fixed-layer definition of the zigzag function. The shear modulus of the fixed layer thus serves as a single weighting coefficient for the entire shear strain energy, thus producing a bias toward the shear stiffness of the fixed layer. In contrast, $\phi_{D S}^{(k)}$ depends on all shear moduli, $G_{x z}^{(k)}$, and ply thicknesses, $2 h^{(k)}$. Its key property is that it vanishes identically when the transverse shear properties are homogeneous, in which case the theory reverts to the underlying shear-deformation theory. The improvements contributed toward solutions for the axial strain, stress, and energy due to the $\phi_{D S}^{(k)} \psi$ term in Equation (1) are particularly appreciable for thick and highly heterogeneous laminates (refer to Section 4).

Di Sciuva's theory runs into further theoretical difficulties in an attempt to interpret the consequences of the shear stress continuity constraints and the resulting uniform shear stress. On the one hand, the correct shear force $V_{x}(x)$ can be determined from the well-established shear-moment relationship

$$
V_{x}=M_{x, x}
$$

which has its origin in the virtual work principle. On the other hand, the shear stress in Equation (12), when integrated over the cross-section, does not yield the correct shear force 


$$
V_{x} \neq \int_{A} \tau_{x z}^{(k)} d A=\tau_{x z}^{(1)} A
$$

A related theoretical anomaly is immediately apparent at a clamped support condition for which the variationally consistent displacement boundary conditions are given as

$$
u=w=w_{, x}=\psi=0
$$

Because $\psi=0$ is required at the clamped end, the corresponding transverse shear strain and stress defined in Equation (9) vanish identically. In contrast, a non vanishing average shear stress $\tau_{x z}=V_{x} / A$ at a clamped end is computed from the shear force obtained from the equilibrium equation given by Equation (13).

From the perspective of finite element approximations appropriate for this theory, $w(x)$ needs to be at least $C^{1}$ continuous, since the axial strain is proportional to the second derivative of $w(x)$. This requirement yields an added impediment for the approximating functions, particularly for developing efficient plate and shell elements based on this class of theory. It has been shown, however, that $C^{0}$ continuous kinematic approximations, usually associated with shear deformation theories of Timoshenko ${ }^{1}$, Mindlin ${ }^{19}$ and Reissner ${ }^{20}$, result in simpler, computationally more efficient, and better performing finite elements than comparable elements based on $C^{1}$ continuous interpolations ${ }^{23}$. It is this aspect that motivated Averill ${ }^{14}$ to introduce a zigzag model based upon the standard form of Timoshenko theory that uses an independent bending rotation variable in the axial displacement expansion.

\subsection{Averill's Zigzag Model}

Averill ${ }^{14}$ proposed a penalty formulation, herein referred to as a Penalized Zigzag (PZ) theory, 
using the standard Timoshenko kinematics as an underlying theory. Consistent with the definition of the fixed zigzag function within the second layer $(k=2)$, the axial displacement of the $P Z$ theory may be expressed as

$$
u_{x}^{(k)}(x, z)=u(x)+\left(z+h-2 h^{(1)}\right) \theta(x)+\phi_{A}^{(k)}(z) \psi(x)
$$

where within this specific example, $\theta(x)$ represents the rotation of the second layer, $u_{x, z}^{(2)}=\theta$, and $\phi_{A}^{(k)}$ denotes Averill's zigzag function depicted in Figure 3(b).

Shear stress continuity conditions, Equation (10), are enforced explicitly only on the first part of the shear stress, Equation (6), by setting $G_{A} \equiv G_{x z}^{(k)}\left(1+\beta^{(k)}\right)$ to be constant across the material layers. Herein, since the second layer is fixed in the sense of the zigzag function, $\phi_{A}^{(2)}=\beta_{A}^{(2)}=0$, thus $G_{A}=G_{x z}^{(2)}$ and the shear stress reduces to

$$
\tau_{x z}^{(k)}=G_{x z}^{(2)} \gamma-G_{x z}^{(k)} \beta_{A}^{(k)} \eta \quad(k=1, \ldots, N)
$$

where $\gamma=\gamma_{x z}^{(2)}$ defines the shear strain in the second (or fixed) layer. Further, the second term in Equation (17) is set to diminish to zero in a limiting sense by letting

$$
\eta \equiv \gamma-\psi \rightarrow 0
$$

with the aid of a penalty constraint term which is added to the strain energy

$$
I_{\lambda} \equiv \frac{\lambda_{A}}{2} \int_{x_{a}}^{x_{b}} \eta^{2} d x
$$


In the above definition, $\lambda_{A}$ represents a penalty factor (units of force) which is set to a large value $\left(\lambda_{A} \rightarrow \infty\right)$ to ensure the validity of Equation (18). The subscript $(\cdot)_{A}$ denotes quantities corresponding to Averill's model. Under these conditions, the shear stresses are uniform only in a limiting sense

$$
\tau_{x z}^{(k)} \rightarrow \tau_{x z}^{(k+1)} \rightarrow G_{x z}^{(2)} \gamma \quad(k=1, \ldots, N-1)
$$

For this theory, the shear force derived from the virtual work principle is given as

$$
V_{x} \equiv \int_{A} \tau_{x z}^{(k)} d A+\lambda_{A} \eta(x)
$$

When the penalty parameter $\lambda_{A} \rightarrow \infty, \eta=O\left(1 / \lambda_{A}\right) \rightarrow 0$ and the term $\lambda_{A} \eta(x)$ takes on a finite value; thus, the shear force in Equation (21), which is derived from the virtual work principle, does not correspond to the cross-sectional integral of the shear stress. Whereas Averill's model results in a four-variable theory and $C^{0}$ continuous kinematics suitable for efficient finite element interpolations, the theory appears to possess some theoretical anomalies. As in Di Sciuva's model, there is the fixedlayer anomaly manifesting itself with a bias toward the shear properties of the fixed layer. This means that the methodology lacks invariance with respect to the choice of the fixed layer. Moreover, the theory breaks down at the clamped end. Because the clamped boundary conditions require the vanishing of the four kinematic variables

$$
u=w=\theta=\psi=0
$$

and the penalization condition Equation (18) yields an additional constraint $w_{, x} \rightarrow 0$, the following erroneous conditions for the shear strain and stress at the clamped end result 


$$
\left(\gamma_{x z}^{(k)}, \tau_{x z}^{(k)}\right)_{\substack{@ \text { clamped } \\ \text { support }}} \rightarrow 0
$$

Averill recognized the above anomaly and proposed to avoid prescribing boundary conditions on the $\psi$ variable, thus violating the theory's variational requirements.

The theoretical difficulties just discussed, associated with the Di Sciuva ${ }^{7}$ and Averill ${ }^{14}$ zigzag theories, serve as motivation for developing a consistent shear deformable zigzag theory that incorporates the proper zigzag kinematics which is devoid of the above mentioned anomalies. In what follows, the mathematical foundation of the new theory, henceforth referred to as the refined zigzag theory, is described.

\section{REFINED ZIGZAG THEORY}

Within the context of the present refined zigzag theory, a zigzag axial-displacement term is superposed onto the Timoshenko kinematic assumptions as in Equation (1), resulting in the strain and stress definitions expressed in Equations (2)-(6). The key differences between the predecessor theories are described and variationally consistent equilibrium equations and boundary conditions are derived from the virtual work principle.

\subsection{Zigzag Function}

The key aspect differentiating the present zigzag function from the previous zigzag models is that it is set to vanish on the outer surfaces of the laminate (refer to Figure 4). This ensures a non-vanishing zigzag distribution through the thickness and, hence, the function contributes to the local distortion of the beam's cross-section due to every layer. 
The axial displacement of Equation (1) may now be interpreted as a superposition of the $u(x)$ and $z \theta(x)$ displacements, which define the bottom and top axial displacements as

$$
u_{x}^{(b)}=u(x)-h \theta(x) \text { and } u_{x}^{(t)}=u(x)+h \theta(x)
$$

and the zigzag component, $\phi^{(k)} \psi(x)$, which permits the distortion of the cross-section within the interior of the laminate such that

$$
\phi^{(k)} \equiv \phi\left(z, u^{(k)}\right)(k=1, \ldots, N-1)
$$

The above definition of the zigzag function implies its dependence upon the interior interface displacements only (refer to Figure 2(b)). Such kinematic arrangement also ensures standard Timoshenko-theory expressions of the axial surface strains

$$
\varepsilon_{x}^{(b)}=u_{, x}-h \theta_{, x} \text { and } \varepsilon_{x}^{(t)}=u_{, x}+h \theta_{, x}
$$

The $\phi^{(k)}$ zigzag function may be conveniently defined in terms of the local (layer) thickness coordinate $\xi^{(k)} \in[-1,1] \quad(k=1, \ldots, N)$ and the normalized $u^{(k)}$ displacements defined along the layer interfaces as

$$
\phi^{(k)} \equiv \frac{1}{2}\left(1-\xi^{(k)}\right) u^{(k-1)}+\frac{1}{2}\left(1+\xi^{(k)}\right) u^{(k)}
$$

where the total interface displacements due to the zigzag term in Equation (1) are defined as $u^{(k)} \psi(x)$. Note that in the present definition, the bottom and top interfacial displacements are set at $u^{(0)}=u^{(N)}=0$, and the local thickness coordinates are defined in terms of the laminate thickness 
coordinate $z \in[-h, h]$ as

$$
\xi^{(k)}=\frac{z-\left(z^{(k-1)}+h^{(k)}\right)}{h^{(k)}} \quad\left(z^{(0)}=-h, \quad z^{(k)}=z^{(k-1)}+2 h^{(k)}\right)
$$

The resulting $\beta^{(k)} \equiv \phi_{, z}^{(k)}$ function is piecewise constant and may be expressed as

$$
\beta^{(k)}=\frac{u^{(k)}-u^{(k-1)}}{2 h^{(k)}}
$$

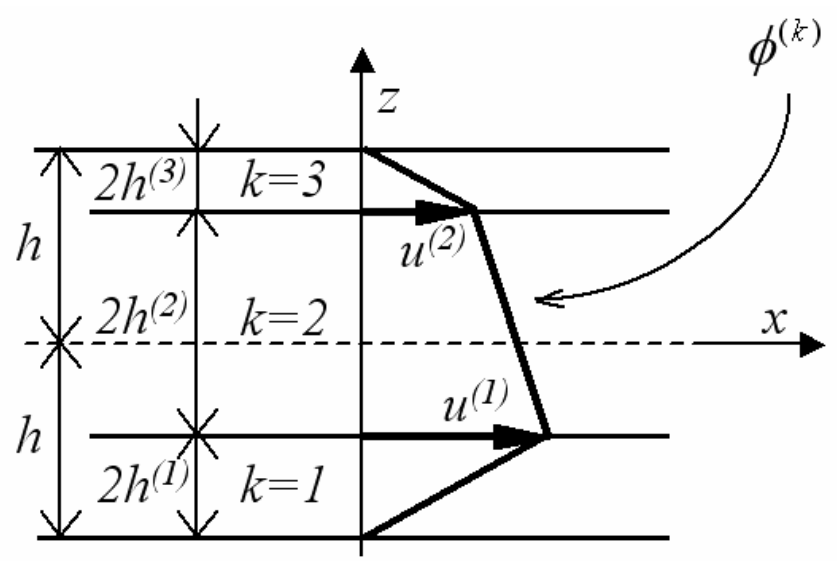

Figure 4. Layer notation for a three-layered laminate and zigzag function of refined zigzag theory.

To determine the unknown interface displacements $u^{(k)}(k=1, \ldots, N-1)$ and thus fully define $\phi^{(k)}$ and $\beta^{(k)}$, the shear stress equilibrium along the interior layer interfaces is not enforced. Instead, the shear coefficient, $G_{x z}^{(k)}\left(1+\beta^{(k)}\right)$, which multiplies the $\gamma$ term in Equation (6) is set to be constant for each layer in the laminate, i.e.,

$$
G \equiv G_{x z}^{(k)}\left(1+\beta^{(k)}\right) \quad(\text { constant })
$$

or, equivalently, the following linear constraint equations are established 


$$
G_{x z}^{(k)}\left(1+\beta^{(k)}\right)=G_{x z}^{(k+1)}\left(1+\beta^{(k+1)}\right) \quad(k=1, \ldots, N-1)
$$

The above constraint strategy is fully consistent with Di Sciuva’s shear-stress continuity constraint as evident from Equations (9) and (10). In Di Sciuva’s case, however, these constraints lead to the full enforcement of shear stress continuity; whereas in the present setting, because of an additional kinematic variable, $\eta$, the condition (30) does not enforce the interface shear-stress continuity.

Thus the shear strain can be expressed in terms of a pair of variables $(\gamma$ and $\psi)$ or $(\gamma$ and $\eta)$ according to

$$
\begin{aligned}
\gamma_{x z}^{(k)} & =\gamma+\left(G / G_{x z}^{(k)}-1\right) \psi \\
& \equiv \frac{1}{G_{x z}^{(k)}}\left[G \gamma+\left(G_{x z}^{(k)}-G\right) \eta\right]
\end{aligned}
$$

It is apparent that the equivalence statements Equation (31) are a means of altering the shear strain definition which is now defined by Equation (32).

Invoking Hooke’s relations, Equation (3), the shear stress takes on the form

$$
\begin{aligned}
\tau_{x z}^{(k)} & =G \gamma+\left(G_{x z}^{(k)}-G\right) \eta \\
& \equiv G \psi+G_{x z}^{(k)} \eta
\end{aligned}
$$

The $\phi^{(k)}$ function thus determined has several unique and desirable properties. First, it gives rise to a particularly useful property for $\beta^{(k)}$ that is true regardless of the layer material properties and thicknesses; that is, 


$$
\int_{A} \beta^{(k)} d A=0
$$

The usefulness of Equation (34) is seen by integrating Equation (5) over the beam cross-section. Performing this process and then recognizing the above $\beta^{(k)}$ property, gives

$$
\gamma=\frac{1}{A} \int_{A} \gamma_{x z}^{(k)} d A
$$

This equation verifies that $\gamma(x)$ is the shear angle of Timoshenko theory and represents an average shear strain of the cross-section. Another useful application of Equation (34) is found by multiplying both sides of Equation (32) by $\beta^{(k)}$, and then integrating over the cross-sectional area. This process reveals that the function $\psi(x)$ is a weighted-average shear strain quantity given by

$$
\psi=\frac{\int_{A} \beta^{(k)} \gamma_{x z}^{(k)} d A}{\int_{A}\left(\beta^{(k)}\right)^{2} d A}
$$

With the homogeneous surface values $\left(u^{(0)}=u^{(N)}=0\right)$ defined a priori, Equations (29) and (30) yield simple and readily computed expressions for the interior interface displacements defining the $\phi^{(k)}$ zigzag function

$$
u^{(k)}=u^{(k-1)}+2 h^{(k)} \beta^{(k)}, \quad \beta^{(k)}=G / G_{x z}^{(k)}-1 \quad(k=1, \ldots, N-1)
$$

Substituting $\beta^{(k)}$ from Equation (37) into Equation (34), the average shear modulus $G$ is readily computed in terms of the layer shear moduli and thicknesses 


$$
G=\left(\frac{1}{A} \int_{A} \frac{d A}{G_{x z}^{(k)}}\right)^{-1} \equiv\left(\frac{1}{h} \sum_{k=1}^{N} \frac{h^{(k)}}{G_{x z}^{(k)}}\right)^{-1}
$$

An alternative procedure that determines the same $\phi^{(k)}$ function is based on the minimization of the shear strain energy ${ }^{17}$.

\subsection{Constant Stress Requirement and Penalization Issues}

In this section, two key issues concerning the validity and technical difficulties of the earlier zigzag concepts are addressed. These are: (1) Explicit enforcement of constant shear stress across beam's cross-section, and (2) Penalty enforcement, in Averill's model, of $\eta \equiv \gamma-\psi \rightarrow 0$, the condition that engenders a theoretical anomaly at the clamped boundary.

Attempts by Di Sciuva ${ }^{7}$ and Averill ${ }^{14}$ have demonstrated that enforcing a constant shear stress across a cross-section, whether explicitly or as a limiting condition, causes various degrees of theoretical anomalies especially manifested at the clamped boundary. Moreover, the issue of penalization of the $\eta$ function has been resolved unsatisfactorily ${ }^{14}$, with an ad hoc penalty term, Equation (18), added to the strain energy of the beam.

It is asserted herein that the failure to accommodate a constant stress condition within the low-order kinematics defined in Equation (1) is due to imposition of excessive constraints that yield non physical conditions. Moreover, a constant shear stress is generally a poor approximation of the exact behaviour for heterogeneous cross-sections. Higher-order approximations, such as the cubic zigzag models $^{12}$, offer greater kinematic freedom, enabling a continuous, non-constant shear stress.

Herein, no attempt will be made to set the term associated with the $\eta$ variable to zero in Equation (33). As will be demonstrated by analytic solutions, the present form of the shear stress is perfectly suited for this class of bending theory.

It is important to point out that the penalization issue of the $\eta$ function can be properly resolved at 
a constitutive equation level as opposed to adding an ad hoc penalty functional to the variational principle $^{14}$. Thus, one can introduce the dimensionless penalty factor, $\lambda_{0}$, in the transverse shear stress constitutive relation associated with the $\eta$ term

$$
\tau_{x z(\lambda)}^{(k)} \equiv G \gamma+\lambda_{0}\left(G_{x z}^{(k)}-G\right) \eta
$$

where, for the special case $\lambda_{0}=1$, Equation (39) reverts back to Equation (33).

The shear strain energy associated with the above shear stress relation is given by

$$
U_{(\lambda)}^{\text {shear }} \equiv \frac{1}{2} \int_{x_{a}}^{x_{b}} \int_{A} \tau_{x z_{(\lambda)}}^{(k)} \gamma_{x z}^{(k)} d A d x=\frac{G A}{2} \int_{x_{a}}^{x_{b}} \gamma^{2} d x+\frac{\lambda}{2} \int_{x_{a}}^{x_{b}} \eta^{2} d x
$$

where $\lambda$ denotes a penalty parameter (of units of force) defined as

$$
\lambda \equiv \lambda_{0} \int_{A} G_{x z}^{(k)}\left(\beta^{(k)}\right)^{2} d A=\lambda_{0}\left(\int_{A} G_{x z}^{(k)} d A-G A\right)
$$

The first term in the shear strain energy has the same exact form as the shear strain energy in Timoshenko theory for a homogeneous beam provided $G$ is the beam's shear modulus. The second term is associated with the $\lambda$ penalty parameter. The term appears naturally as a consequence of the modified constitutive relations. For homogeneous cross-sections, $\lambda$ vanishes due to $\beta^{(k)}=0$ and $G$ becomes the beam's shear modulus. This is contrasted with Averill's penalization, where a similar penalty term is added to the strain energy a posteriori. The inclusion of the penalty factor $\lambda_{0}$ under the condition $\lambda_{0} \rightarrow \infty$ allows for the limiting condition, Equation (18), to be fulfilled. Also note that the coupling term $\gamma \eta$ drops out from the shear energy due to the intrinsic property of the present 
zigzag function manifested by Equation (34).

The fully consistent form of the present theory $\left(\lambda_{0}=1\right)$ does not enforce condition (18); hence, its shear stress and strain are piecewise constant across the laminated cross-section, and no anomalies are encountered at a clamped boundary. The same basic conclusions may be reached for any arbitrary value of $\lambda_{0}$, including the very large values. In this case, $\eta=O\left(\frac{1}{\lambda_{0}}\right)$ and even for $\lambda_{0} \rightarrow \infty$, the second term of the shear stress in Equation (39) does not vanish and is on the order of $O\left(G-G_{x z}^{(k)}\right)$.

\subsection{Virtual Work Principle, Equilibrium Equations, and Boundary Conditions}

For the beam defined in Figure 1, the virtual work principle (VWP) may be expressed as

$$
\begin{aligned}
& \int_{x_{a}}^{x_{b}} \int_{A}\left(\sigma_{x}^{(k)} \delta \varepsilon_{x}^{(k)}+\tau_{x z_{(\lambda)}^{(k)}} \delta \gamma_{x z}^{(k)}\right) d A d x-\int_{x_{a}}^{x_{b}} q \delta w d x \\
& +\int_{A}\left[T_{x a} \delta u_{x}^{(k)}\left(x_{a}, z\right)+T_{z a} \delta w\left(x_{a}\right)\right] d A-\int_{A}\left[T_{x b} \delta u_{x}^{(k)}\left(x_{b}, z\right)+T_{z b} \delta w\left(x_{b}\right)\right] d A=0
\end{aligned}
$$

Performing the cross-sectional integration and variation by parts, gives rise to the following Euler equilibrium equations

$$
\begin{array}{r}
N_{x, x}=0 \\
M_{x, x}-V_{x}=0 \\
V_{x, x}+q=0 \\
M_{\phi, x}-V_{\phi}=0
\end{array}
$$

and a consistent set of boundary conditions 


$$
\begin{array}{lllc}
\text { either } & u\left(x_{\alpha}\right)=\bar{u}_{\alpha} & \text { or } & N_{x}\left(x_{\alpha}\right)=\bar{N}_{x \alpha} \\
\text { either } & \theta\left(x_{\alpha}\right)=\bar{\theta}_{\alpha} & \text { or } & M_{x}\left(x_{\alpha}\right)=\bar{M}_{x \alpha} \\
\text { either } & w\left(x_{\alpha}\right)=\bar{w}_{\alpha} & \text { or } & V_{x}\left(x_{\alpha}\right)=\bar{V}_{x \alpha} \\
\text { either } & \psi\left(x_{\alpha}\right)=\bar{\psi}_{\alpha} & \text { or } & M_{\phi}\left(x_{\alpha}\right)=\bar{M}_{\phi \alpha}
\end{array}
$$

where $\alpha=a, b$ and the bar-superscripted symbols denote the prescribed displacements and stress resultants. The first three equilibrium equations in Equation (43) have the same form as those found in classical and Timoshenko theories, whereas the fourth equation describes the moment-shear equilibrium involving the zigzag-related bending moment and shear force. The following crosssectional area integrals represent the reactive and prescribed stress resultants

$$
\begin{aligned}
& \left(N_{x}, \quad M_{x}, \quad M_{\phi}, \quad V_{x}, \quad V_{\phi}\right) \equiv \int_{A}\left(\sigma_{x}^{(k)}, \quad z \sigma_{x}^{(k)}, \quad \phi^{(k)} \sigma_{x}^{(k)}, \quad \tau_{x z}^{(k)}, \quad \beta^{(k)} \tau_{x z}^{(k)}\right) d A \\
& \left(\begin{array}{llll}
\bar{N}_{x \alpha}, & \bar{M}_{x \alpha}, & \bar{M}_{\phi \alpha}, & \bar{V}_{x \alpha}
\end{array}\right) \equiv \int_{A}\left(T_{x \alpha}, \quad z T_{x \alpha}, \quad \phi^{(k)} T_{x \alpha}, \quad T_{z \alpha}\right) d A
\end{aligned}
$$

where $N_{x}, M_{x}$ and $V_{x}$ are respectively the conventional axial force, bending moment, and shear force; $M_{\phi}$ and $V_{\phi}$ are the bending moment and shear force due to the zigzag distortion of the beam's crosssection.

Integrating Equation (45) results in the constitutive relations of the beam theory

$$
\left\{\begin{array}{l}
N_{x} \\
M_{x} \\
M_{\phi}
\end{array}\right\}=\left[\begin{array}{lll}
A_{11} & B_{12} & B_{3} \\
B_{12} & D_{11} & D_{12} \\
B_{13} & D_{12} & D_{22}
\end{array}\right]\left\{\begin{array}{c}
u_{, x} \\
\theta_{, x} \\
\psi_{, x}
\end{array}\right\}
$$

where

$$
\left(A_{11}, B_{12}, D_{11}\right) \equiv \int_{A} E_{x}^{(k)}\left(1, z, z^{2}\right) d A, \quad\left(B_{13}, D_{12}, D_{22}\right) \equiv \int_{A} E_{x}^{(k)} \phi^{(k)}\left(1, z, \phi^{(k)}\right) d A
$$




$$
\left\{\begin{array}{c}
V_{x} \\
V_{\phi}
\end{array}\right\}=\left[\begin{array}{ll}
Q_{11}^{2} & Q_{12}^{2} \\
Q_{12}^{\imath} & Q_{22}^{2}
\end{array}\right]\left\{\begin{array}{c}
\theta+w_{, x} \\
\psi
\end{array}\right\}
$$

where

$$
\left(Q_{11}^{\lambda}, Q_{12}^{\lambda}, Q_{22}^{\lambda}\right) \equiv(Q+\lambda,-\lambda, \lambda), Q \equiv \int_{A} G_{x z}^{(k)}\left(1+\beta^{(k)}\right)^{2} d A \equiv G A
$$

Note that a Timoshenko-type shear correction factor is not used within the present theory when modelling a heterogeneous cross-section. For homogeneous beams or laminated beams in which the shear moduli are the same (or nearly the same), the present theory reduces back to Timoshenko theory. In this case, it is appropriate to use the standard shear correction factor $k^{2}=5 / 6$ (for a rectangular cross-section) in the definition of the shear stress.

Substituting the stress resultants defined in Equations (47) and (48) into Equation (43) yields the equilibrium equations in terms of the kinematic variables of the theory

$$
\begin{aligned}
& A_{11} u_{, x x}+B_{12} \theta_{, x x}+B_{13} \psi_{, x x}=0 \\
& Q_{11}^{\lambda}\left(w_{, x x}+\theta_{, x}\right)+Q_{12}^{\lambda} \psi_{, x}=-q(x) \\
& B_{12} u_{, x x}+D_{11} \theta_{, x x}+D_{12} \psi_{, x x}-Q_{11}^{\lambda}\left(w_{, x}+\theta\right)-Q_{12}^{\lambda} \psi=0 \\
& B_{13} u_{, x x}+D_{12} \theta_{, x x}+D_{22} \psi_{, x x}-Q_{12}^{\lambda}\left(w_{, x}+\theta\right)-Q_{22}^{\imath} \psi=0
\end{aligned}
$$

The solution to the above boundary value problem involves integration of the four equilibrium equations (49), subject to the boundary conditions (44), while obeying the constitutive relations (47) and (48). 


\section{EXAMPLE PROBLEMS}

The predictive capability of the new, refined zigzag beam theory is demonstrated for two beam examples that provide a challenge to the theory for certain laminate constructions and beamslenderness ratios. The first example is a simply supported beam of length $L$ that is subjected to the

sinusoidal transverse line load $q(x)=q_{0} \sin (\pi x / L)$, as shown in Figure 5. The boundary conditions at the ends $x=0$ and $x=L$ of the beam are

$$
x=0, L: \quad w=N_{x}=M_{x}=M_{\phi}=0
$$

The following trigonometric expressions for the four kinematic variables

$$
\begin{aligned}
w(x) & =w_{0} \sin (\pi x / L), \\
(u(x), \quad \theta(x), \quad \psi(x)) & =\left(\begin{array}{lll}
u_{0}, & \theta_{0}, \quad \psi_{0}
\end{array}\right) \cos (\pi x / L)
\end{aligned}
$$

satisfy the above boundary conditions exactly. The amplitudes $w_{0}, u_{0}, \theta_{0}$, and $\psi_{0}$ are uniquely determined by substituting Equation (51) into the equilibrium equations, Equation (49), and then solving the resulting system of four linear algebraic equations.

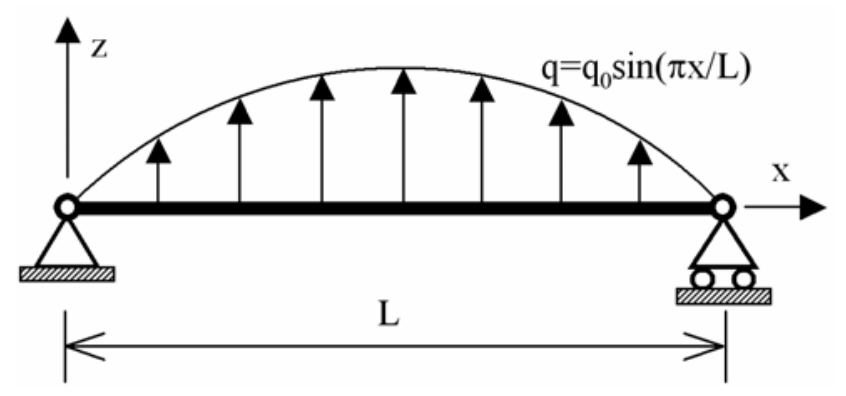

Figure 5. A simply supported beam. 
The second example presented herein is a cantilevered beam of length $L$ that is subjected to a transverse load $\mathrm{F}$ at the free end, as shown in Figure 6. The boundary conditions are given by

$$
\begin{array}{ll}
x=0: & u=w=\theta=\psi=0 \\
x=L: & N_{x}=M_{x}=M_{\phi}=0, \quad V_{x}=F
\end{array}
$$

Because $q(x)=0$ for this problem, the equilibrium equations, Equations (49), are readily simplified, giving rise to the following exact solutions for the kinematic variables

$$
\begin{aligned}
& \psi(x)=a_{1} \cosh (R x)+a_{2} \sinh (R x)+a_{3}, \\
& \theta(x)=\left(A_{1}+\frac{A_{2}}{R^{2}}\right)\left(a_{1} \cosh (R x)+a_{2} \sinh (R x)\right)+\frac{A_{2} a_{3}}{2} x^{2}+a_{4} x+a_{5}, \\
& \begin{aligned}
u(x)= & \left(A_{3}+\frac{A_{4}}{R^{2}}\right)\left(a_{1} \cosh (R x)+a_{2} \sinh (R x)\right)+\frac{A_{4} a_{3}}{2} x^{2}+a_{6} x+a_{7}, \\
w(x) & =\left[A_{6} R+\frac{A_{5}-A_{1}}{R}-\frac{A_{2}}{R^{3}}\right]\left(a_{1} \sinh (R x)+a_{2} \cosh (R x)\right) \\
& -\frac{A_{2} a_{3}}{6} x^{3}-\frac{a_{4}}{2} x^{2}+\left[A_{5} a_{3}-a_{5}\right] x+a_{8}
\end{aligned}
\end{aligned}
$$

where $R=\sqrt{\left|A_{2} / A_{0}\right|}, A_{i}(\mathrm{i}=0, \ldots, 6)$ are quantities that depend on the stiffness coefficients of the model (Equations (47) and (48)) and $a_{i}(i=1, \ldots, 8)$ are unknown constants to be determined from the boundary conditions stated by Eqs. (52). The solution described in Equation (53) is valid for $A_{2} / A_{0}<0$; for $A_{2} / A_{0}>0$, trigonometric functions should be used instead of the hyperbolic functions 17. 


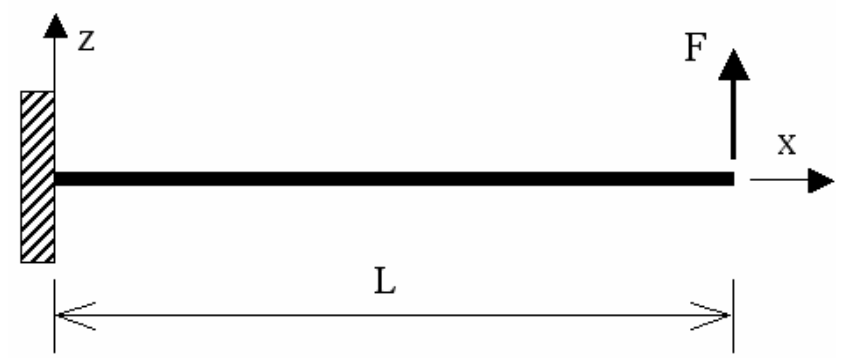

Figure 6. A cantilevered beam.

\section{RESULTS AND DISCUSSION}

Results are presented in this section for the two beam examples previously described. The results include comparisons of those obtained from the new zigzag theory to those obtained using Timoshenko, Di Sciuva and Averill's zigzag theories, exact elasticity solutions, and high-fidelity finite element solutions. A thick, three-layer beam is considered for all problems, with total thickness $2 \mathrm{~h}=2[\mathrm{~cm}]$ and length $\mathrm{L}=10[\mathrm{~cm}]$. The span-to-thickness ratio is $\mathrm{L} / 2 \mathrm{~h}=5$ and each beam has a rectangular cross-section. The mechanical properties of six layer materials that were used to generate results are presented in Table 1 . The major principal axis of each material is aligned parallel to the beam axis. Three unidirectional laminate stacking sequences that were considered are presented in Table 2. The layer thicknesses are presented in the form $\left(2 h^{(1)}, 2 h^{(2)}, 2 h^{(3)}\right)$ and the first layer in each laminate starts at $\mathrm{z}=-\mathrm{h}$. Similarly, the material composition of each layer is presented in the form $\left(M^{(1)}, M^{(2)}, M^{(3)}\right)$, where “ $M$ ” denotes the material type.

\subsection{Reference Solutions}

The following reference solutions, used for the purpose of assessing the predictive capability of the present theory, are briefly described.

1. “Exact” refers to an exact elasticity solution for a simply supported beam ${ }^{21}$.

2. “FEM/NASTRAN” refers to a high-fidelity, two-dimensional FEM solution obtained with the 
MSC/NASTRAN ${ }^{\circledR}$ code. The cantilevered beam is analyzed using a two-dimensional plane-stress model discretized with QUAD8 elements that are based on serendipity shape functions ${ }^{24}$. The discretization involves a uniform mesh having 40 elements in the direction of the beam thickness and 200 along its length, with a total of 8000 elements, and nearly 49,000 degrees-of-freedom ${ }^{17}$.

3. "TBT" refers to the exact Timoshenko Beam Theory solutions. Here, for the sake of consistency, the standard shear correction factor $k^{2}=5 / 6$, which is appropriate for homogeneous, rectangular crosssection beams, is used throughout. Note that various approaches have been proposed to determine lamination-appropriate shear correction factors that commonly provide more accurate deflection predictions. Note that in Timoshenko theory the value of $k^{2}$ influences only the deflection and the transverse shear strain results; however, $k^{2}$ does not affect results for the axial strain and stress, and the shear stress.

4. “Zigzag (D,A)” refers to exact solutions obtained with the previous zigzag theories ${ }^{7,14}$, producing virtually identical results.

5. "Zigzag (R)” refers to the refined zigzag theory solutions obtained with $\lambda_{0}=1$.

6. “Zigzag $\left(\mathrm{R}\left(\lambda_{0}=10^{6}\right)\right)$ ” refers to the refined zigzag theory solutions obtained with $\lambda_{0}=10^{6}$.

7. “Zigzag (All)” refers to nearly identical solutions obtained by all three zigzag models presented herein (Di Sciuva ${ }^{7}$, Averill ${ }^{14}$ and the present refined zigzag theory).

8. "Integrated" refers to the shear stress obtained by integrating the equilibrium equation $\sigma_{x, x}^{(k)}+\tau_{x z, z}^{(k)}=0$, in which $\sigma_{x}^{(k)}$ represents the axial stress determined from the refined zigzag theory $\left(\lambda_{0}=1\right)$. This commonly employed shear-stress recovery method has shown to produce accurate shear stresses $\tau_{x z}^{(k)}$ that are found to be in close agreement with those of the reference FEM model.

In Figs. 7-24, the axial and transverse coordinates are normalized as $(\tilde{x}, \tilde{z}) \equiv(x / L, \quad z / h)$. Similarly, the dimensionless solution variables 


$$
\left(\tilde{u}_{x}^{(k)}, \quad \tilde{u}_{z}^{(k)}\right) \equiv \frac{\pi^{4} D_{11}}{10 q_{0} L^{4}}\left(u_{x}^{(k)}, \quad u_{z}^{(k)}\right), \quad\left(\tilde{\sigma}_{x}^{(k)}, \quad \tilde{\tau}_{x z}^{(k)}\right) \equiv \frac{2 h A \pi^{2}}{q_{0} L^{2}}\left(\sigma_{x}^{(k)}, \quad \tau_{x z}^{(k)}\right)
$$

are used for the simply supported beams, and for the cantilevered beams

$$
\begin{aligned}
& \left(\tilde{u}_{x}^{(k)}, \quad \tilde{u}_{z}^{(k)}, \quad \tilde{w}\right) \equiv \frac{D_{11}}{10 F L^{3}}\left(u_{x}^{(k)}, u_{z}^{(k)}, w\right), \quad\left(\tilde{\sigma}_{x}^{(k)}, \tilde{\tau}_{x z}^{(k)}\right) \equiv \frac{2 h A}{F L}\left(\sigma_{x}^{(k)}, \tau_{x z}^{(k)}\right), \\
& \tilde{V}_{x} \equiv \frac{1}{F} \int_{A} \tau_{x z}^{(k)} d A
\end{aligned}
$$

are used.

\subsection{Results for Simply Supported Beams}

Normalized axial displacements, transverse displacements, and transverse-shear stress distributions are presented in Figures 7-9, respectively, for the simply supported beam made from Laminate A - a three-layer sandwich with a very thick, compliant core. Three curves are shown in Figures 7 and 9 and two curves are shown in Figure 8. The solid and dashed black curves correspond to results from the exact elasticity solution and all three zigzag models presented herein, respectively. The finely dashed gray line corresponds to results from Timoshenko theory. The abscissa in Figure 8 is scaled to exaggerate the difference in the results obtained from the exact elasticity solution and the three zigzag models. As a result, the transverse deflection obtained using Timoshenko theory, $\tilde{u}_{z}(L / 2)=0.164$, is not shown. 


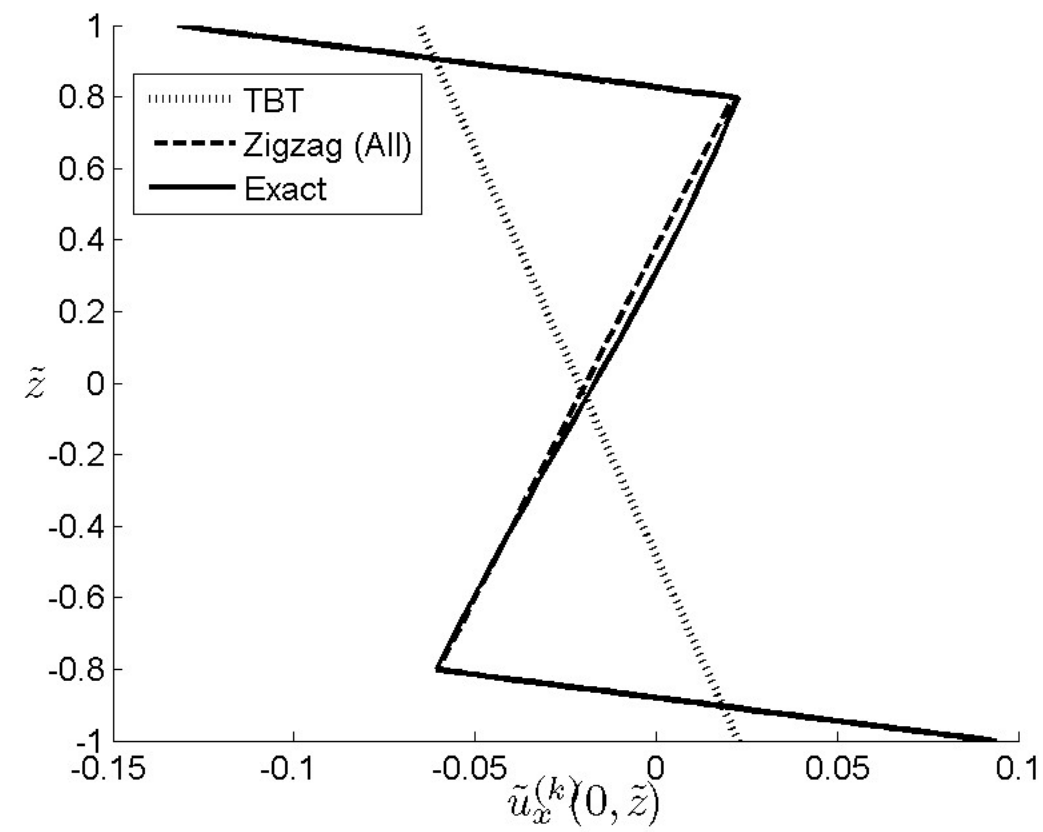

Figure 7. Simply supported beam, laminate A: Normalized axial displacement at left end.

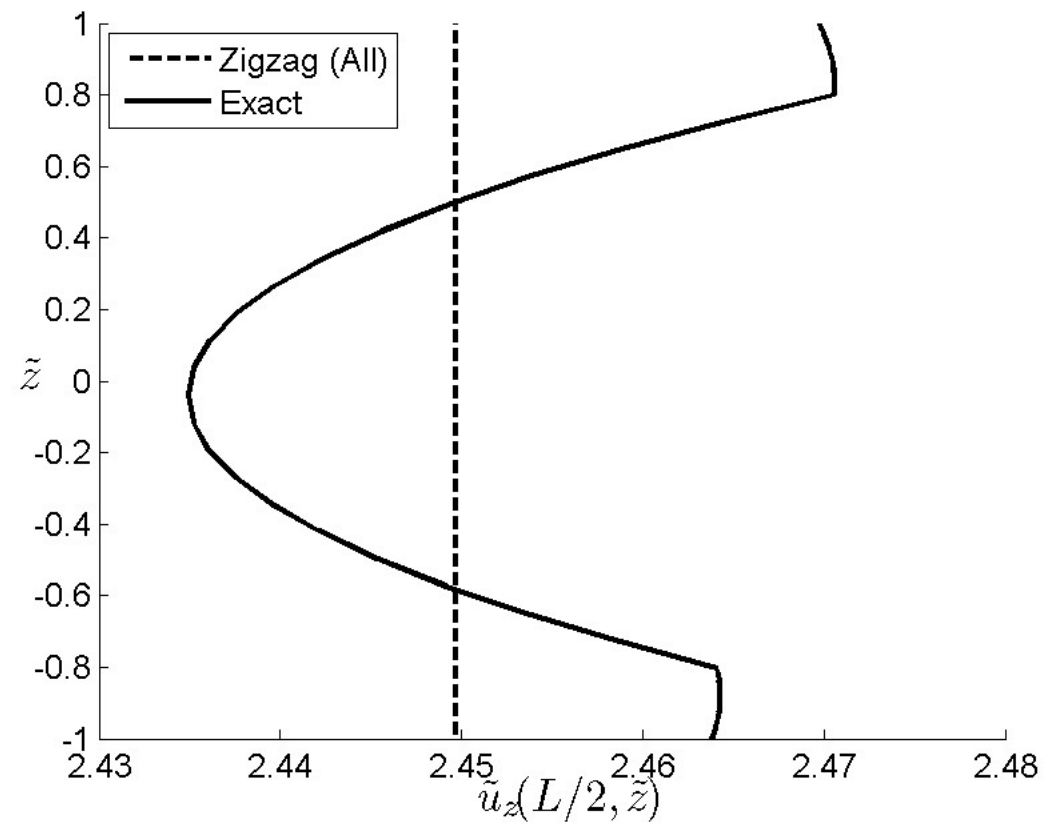

Figure 8. Simply supported beam, laminate A: Normalized transverse displacement at mid-span. (Not shown is the non-dimensional maximum deflection, $\tilde{u}_{z}(L / 2)=0.164$, due to Timoshenko theory.) 


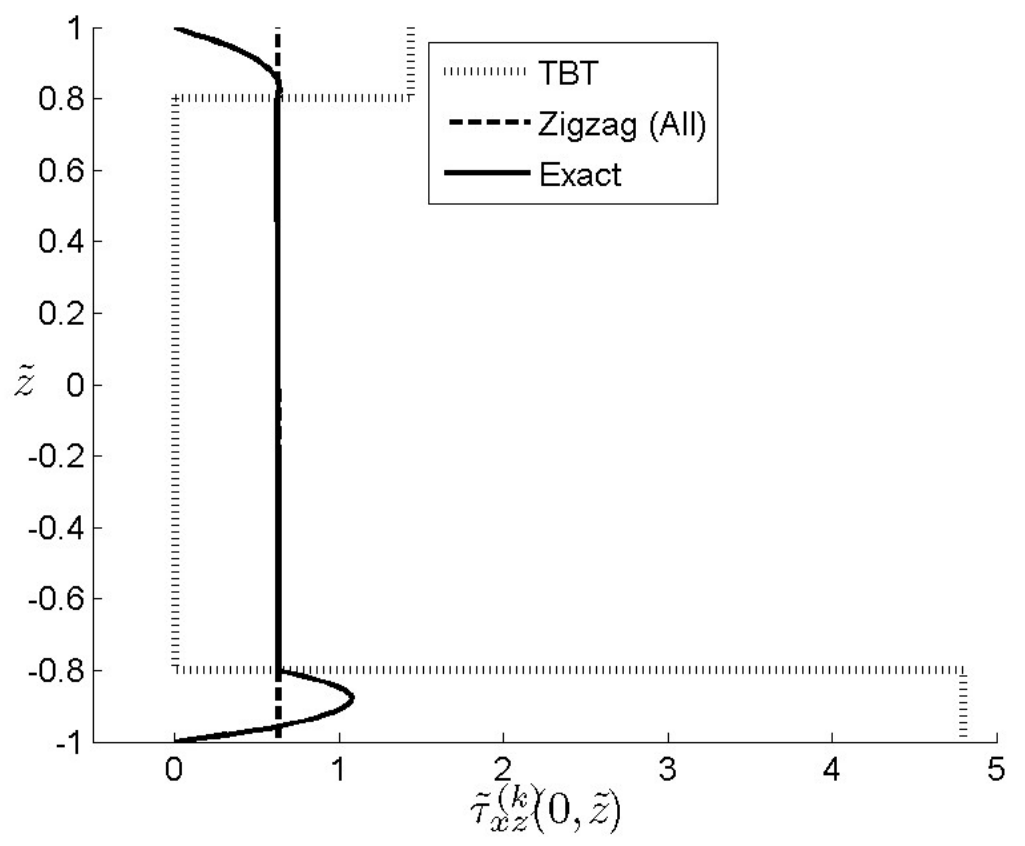

Figure 9. Simply supported beam, laminate A: Normalized transverse shear stress at left end.

The results in Figures 7-9 show that modelling the deformations of Laminate A is a particularly challenging problem for any structural theory. The beam is relatively thick $(\mathrm{L} / 2 \mathrm{~h}=5)$ and has a high degree of anisotropy typical of sandwich construction: a highly compliant thick layer bounded by two relatively stiff, thin face sheets. For this problem, Timoshenko theory under predicts the deflection by a factor of about 15 (Figure 8). All zigzag theories produce comparable displacement and stress results that correlate well with the exact solutions. As seen from Figure 9, only slight differences are observed in the shear stress as predicted by the original and refined zigzag theories, its distribution being respectively constant and piecewise constant. Contrasted with the zigzag results, Timoshenko’s shear stress is substantially under estimated in the core and grossly over estimated in the face sheets. 


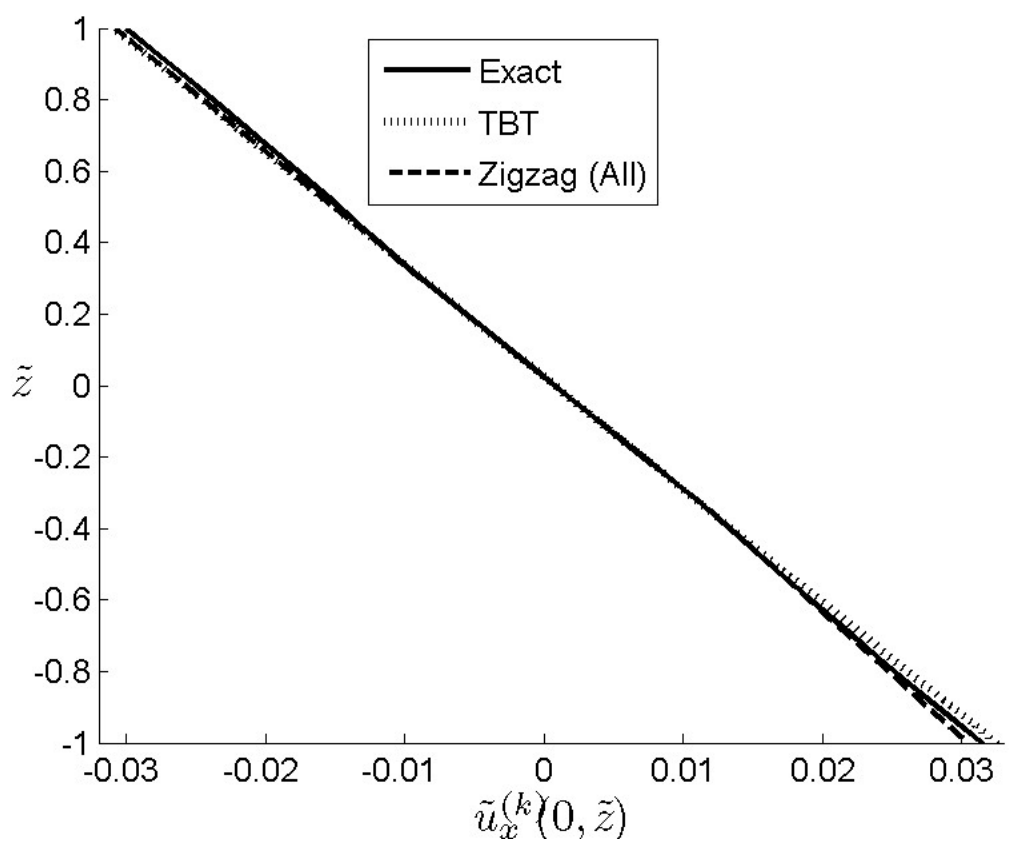

Figure 10. Simply supported beam, laminate C: Normalized axial displacement at left end.

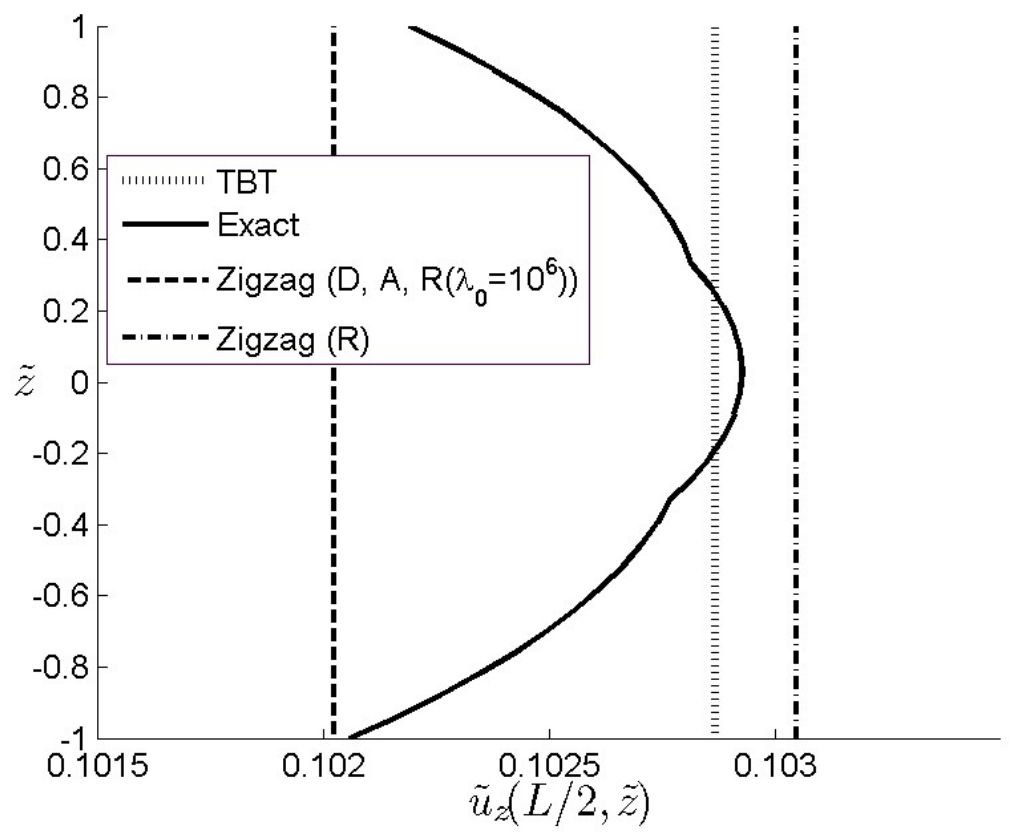

Figure 11. Simply supported beam, laminate C: Normalized transverse displacement at mid-span. 

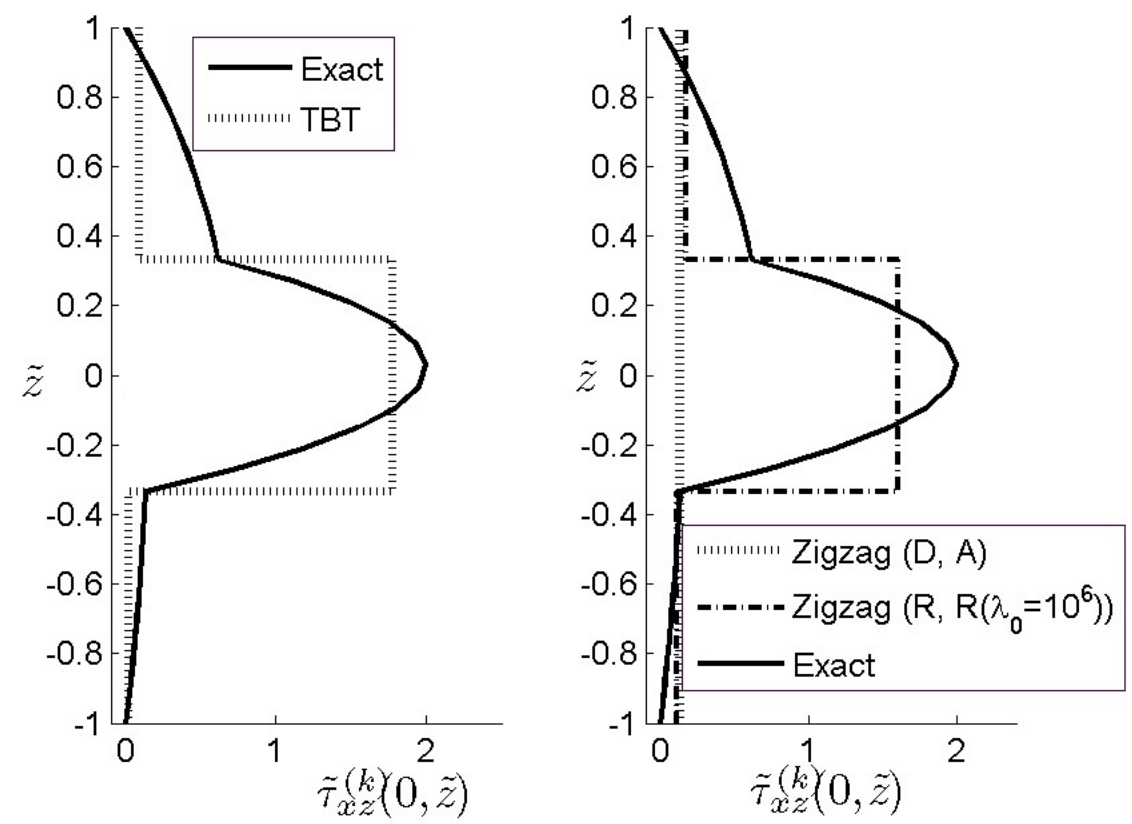

Figure 12. Simply supported beam, laminate C: Normalized transverse shear stress at left end.

Laminate C has three layers of equal thickness, with a very stiff inner layer (Figures 10-12). For this laminate, Timoshenko theory models the overall beam stiffness adequately and produces accurate displacement and transverse shear stress predictions. As shown in Figure 11, the refined zigzag theory, when used with a large penalty factor, $\lambda_{0}=10^{6}$, produces a slightly lower deflection than in the $\lambda_{0}=1$ case, where the latter solution only slightly exceeds the exact value at $\tilde{Z}=0$. Also note that the deflection solution corresponding to the $\lambda_{0}=10^{6}$ case is closely correlated with the original zigzag theory predictions that are somewhat stiffer than those of the refined theory with $\lambda_{0}=1$. As evidenced from Figure 12, the refined zigzag theory demonstrates a notable improvement over the original zigzag theories in the transverse shear stress predictions. This example clearly demonstrates that, even in the simply supported case, the original zigzag theories may produce rather inaccurate shear stresses, even when compared to those of Timoshenko theory. Moreover, the non penalized form $\left(\lambda_{0}=1\right)$ of the refined zigzag theory produces slightly more flexible and accurate results than its penalized $\left(\lambda_{0}=10^{6}\right)$ counterpart. For this reason, subsequent results of the refined zigzag theory are 
only demonstrated for the non-penalized case $\left(\lambda_{0}=1\right)$.

\subsection{Results for Cantilevered Beams}

In this section, normalized displacements, stresses, and shear-stress resultants are presented in Figures 13-24 for the clamped beam made of Laminates A and B.

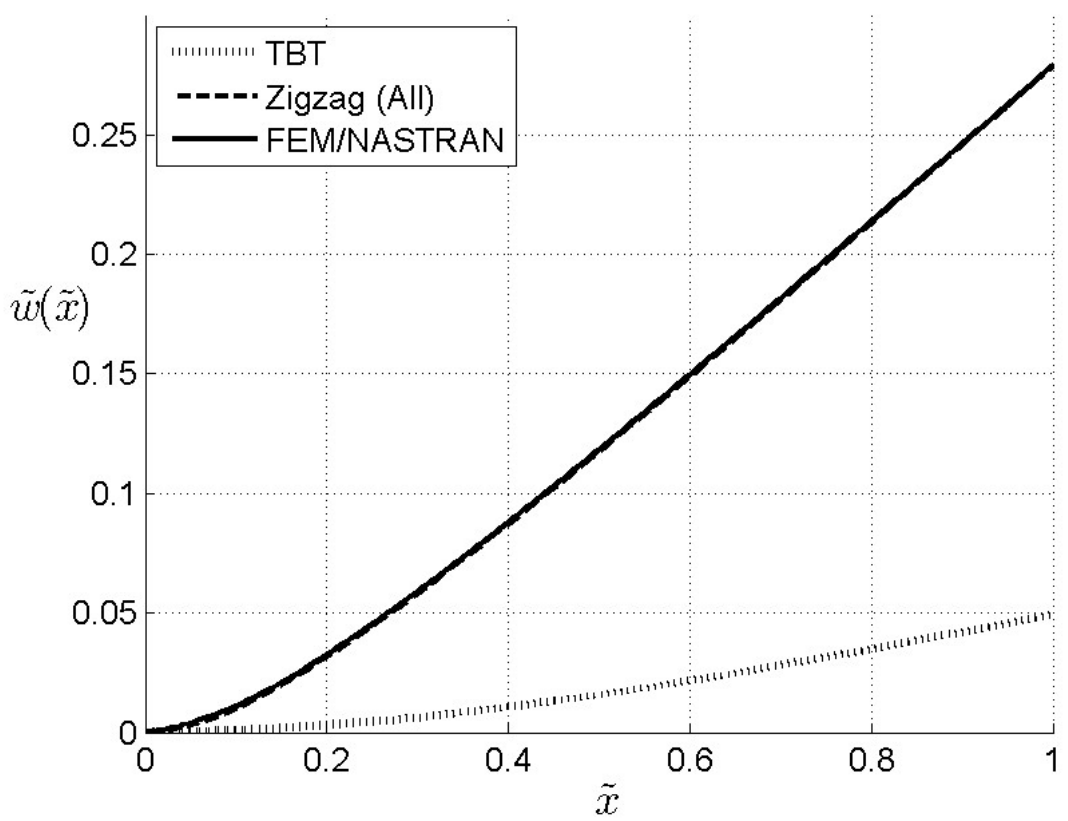

Figure 13. Cantilevered beam, laminate A: Normalized deflection along beam span. 


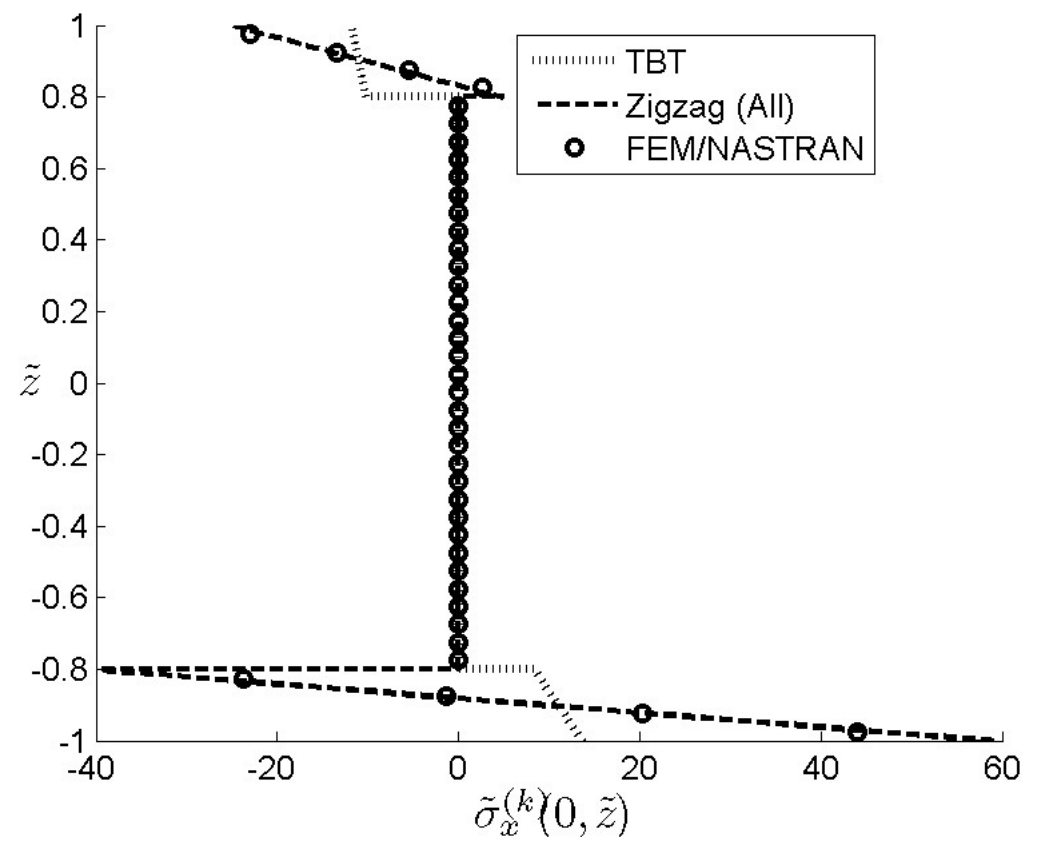

Figure 14. Cantilevered beam, laminate A: Normalized axial stress at clamped end.

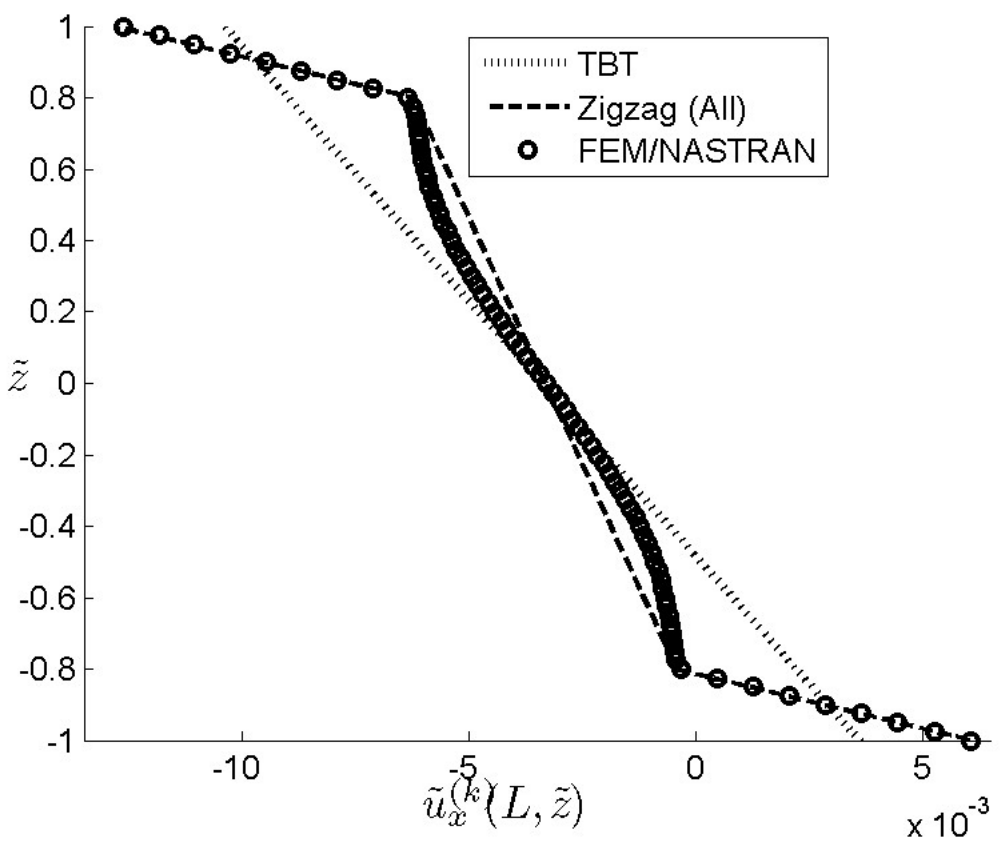

Figure 15. Cantilevered beam, laminate A: Normalized axial displacement at free end. 


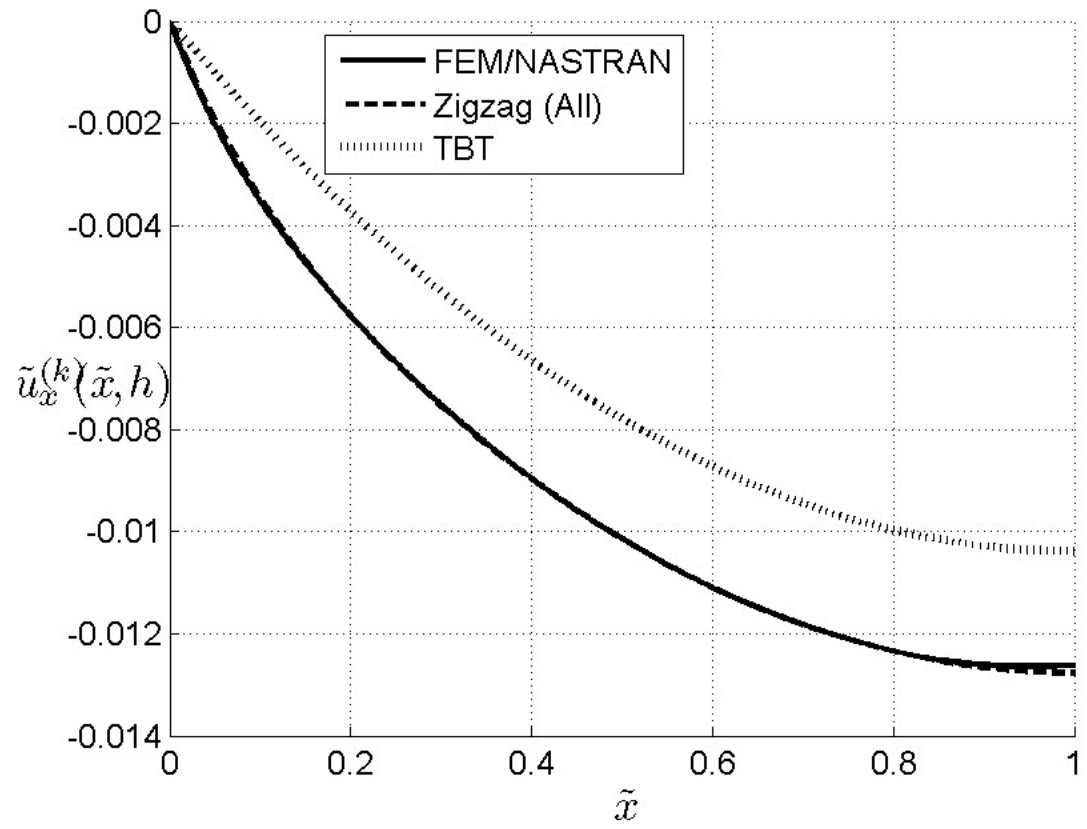

Figure 16. Cantilevered beam, laminate A: Normalized axial displacement along top face of beam.
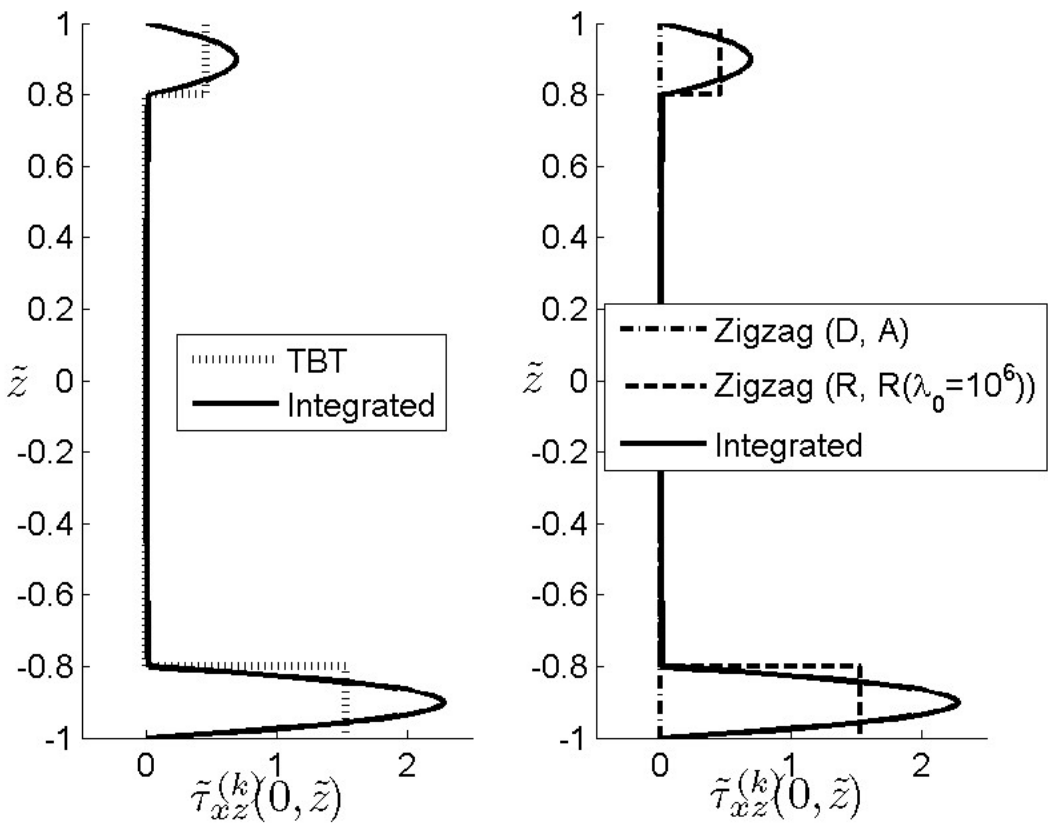

Figure 17. Cantilevered beam, laminate A: Normalized transverse shear stress at clamped end. 

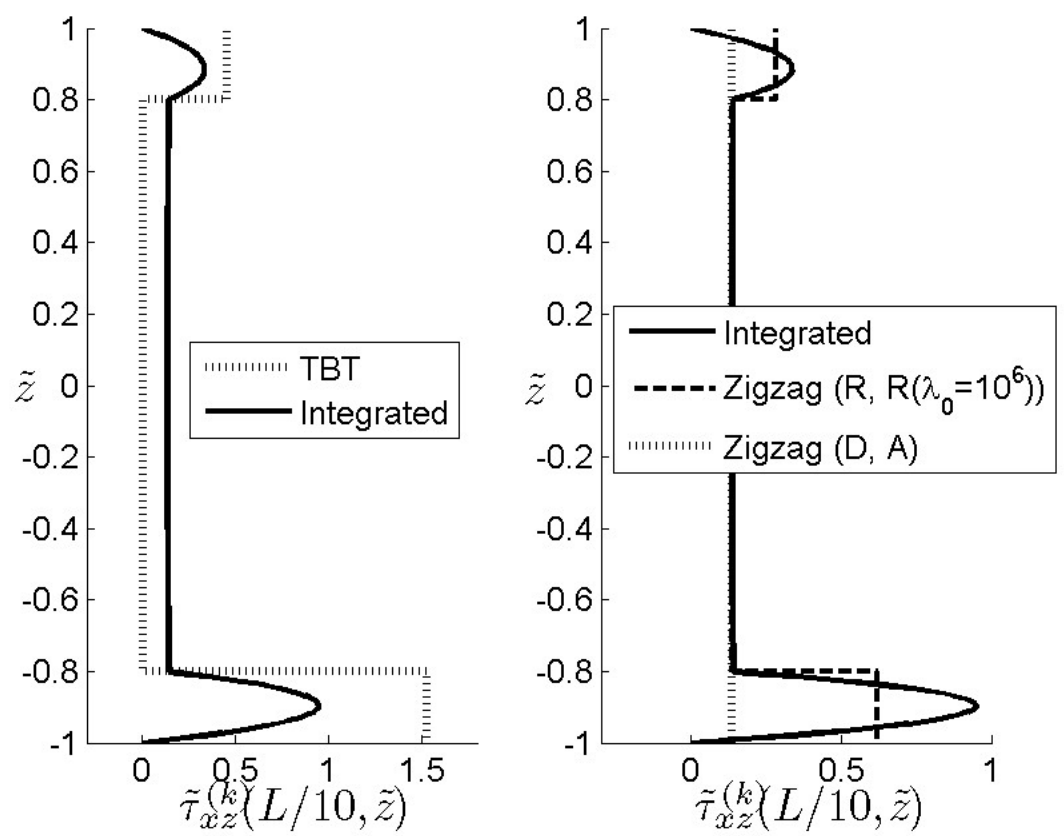

Figure 18. Cantilevered beam, laminate A: Normalized transverse shear stress at $\mathrm{x}=\mathrm{L} / 10$.
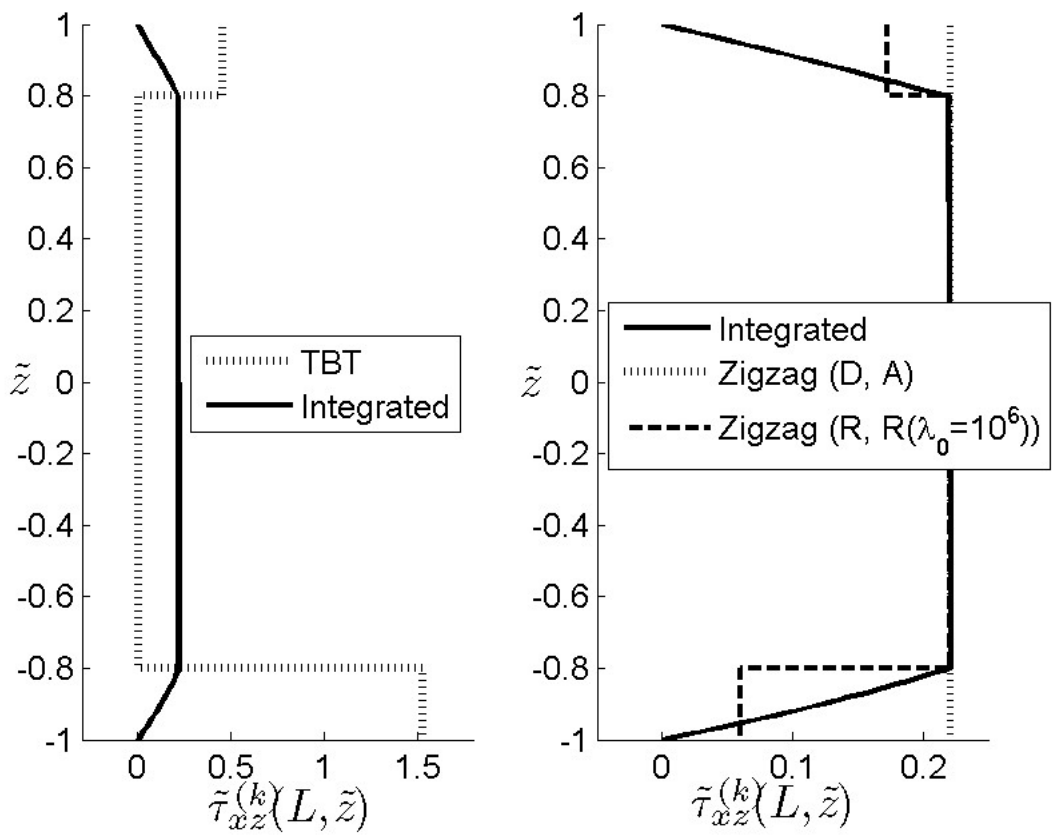

Figure 19. Cantilevered beam, laminate A: Normalized transverse shear stress at free end. 


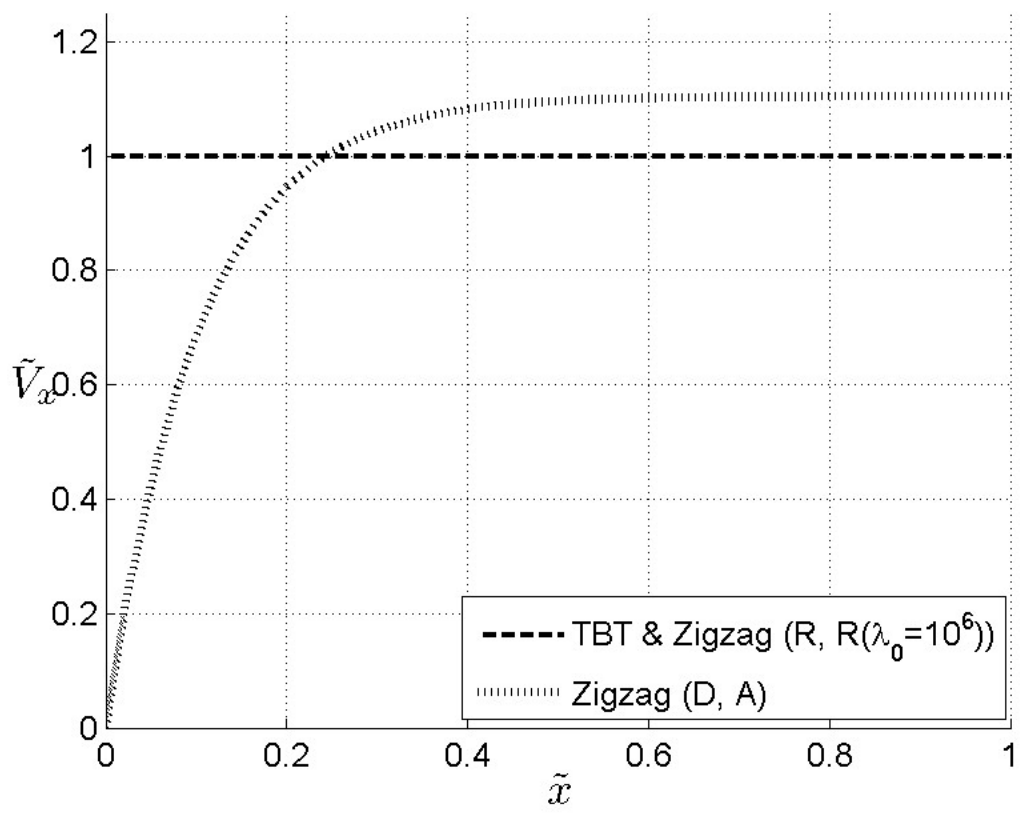

Figure 20. Cantilevered beam, laminate A: Normalized shear force along beam span.

The sandwich-type Laminate $\mathrm{A}$ is again a major challenge for Timoshenko theory that over estimates the over all stiffness of the beam and under estimates the maximum deflection by over $80 \%$ and the maximum axial stress by over 50\% (Figures 13 and 14). These results contrast with those of the zigzag theories that produce very accurate displacements and stresses.

Differences between the various zigzag solutions are observed by way of through-the-depth distributions for the displacements and stresses examined at various locations along the span, including the clamped end. The axial stress and displacement depicted in Figures 14-16 are accurately modelled by all zigzag theories examined, exhibiting only minor quantitative differences. This is attributed to their built-in zigzag kinematics, giving rise to the requisite slope changes at the layer interfaces due to the layer differences in shear moduli.

Examination of the transverse shear stress reveals specific solution differences corresponding to the beam theories examined. At the clamped end (Figures 17), the refined zigzag theory yields a nonvanishing stress, whereas the original zigzag theory produces an erroneous, zero shear stress. Away 
from the clamped end (Figures 18 and 19), the shear stress distributions also exhibit substantial differences. In this problem, the cross-sectional distribution of the shear stress changes significantly along the beam's span, as expressed by the reference and refined zigzag theory solutions. The original zigzag theory produces a shear stress that varies in magnitude with respect to the axial coordinate, while remaining constant across individual cross-sections. The shear stress of the refined zigzag theory also varies with respect to the axial coordinate; however, it is piecewise constant across the cross-sections. In contrast, the shear stress due to Timoshenko theory remains constant with respect to the axial coordinate and exhibits inferior accuracy across the beam’s cross-sections. Finally, the shear force distribution along the beam's span clearly pinpoints the transverse shear anomaly inherent within the original zigzag theory. As shown in Figure 20, the original zigzag theory predicts the vanishing at the clamped end of the normalized shear force quantity, $\tilde{V}_{x}$ (i.e. the normalized integral of the shear stress along the beam cross section, see Equation (55) and Equation (21)). Nearly halfway across the beam, an asymptotic value is achieved that over estimates the correct value by about $10 \%$. Note, however, that a correct shear force distribution corresponding to Di Sciuva’s theory is given by the derivative of the bending moment. Both Timoshenko and the refined zigzag theories give rise to a correct, constant shear force over the entire span of the beam. 


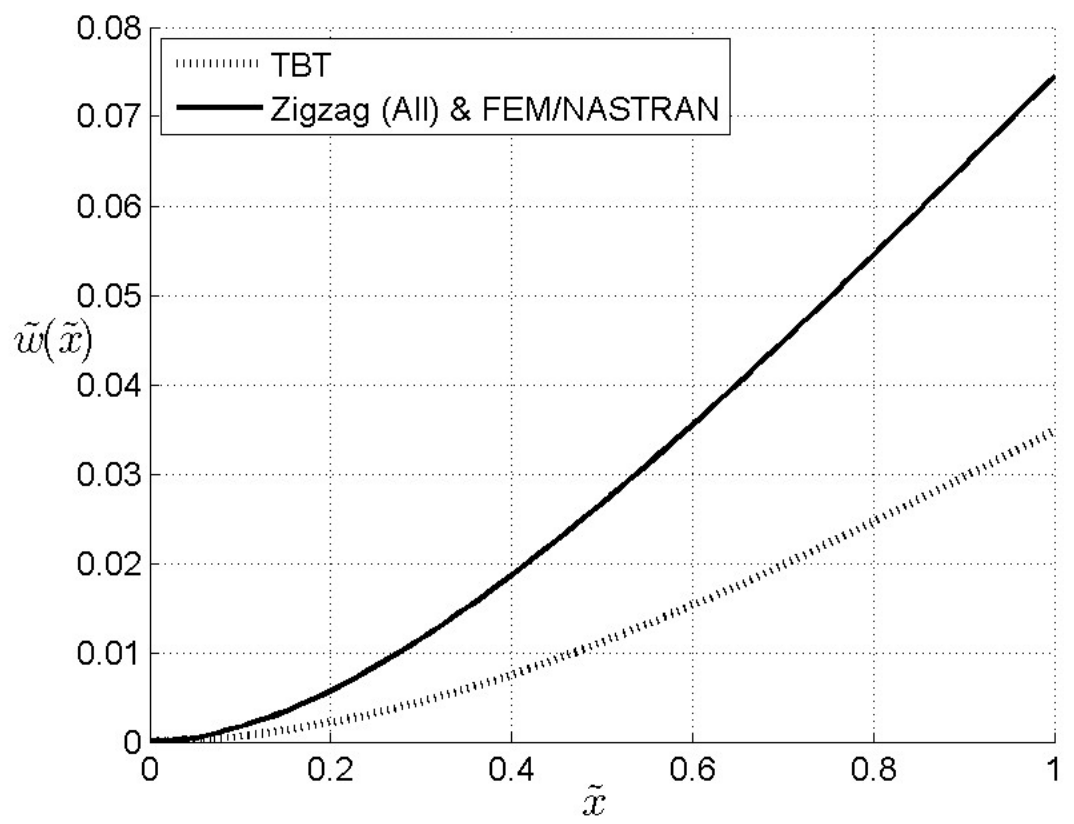

Figure 21. Cantilevered beam, laminate B: Normalized deflection along beam span.
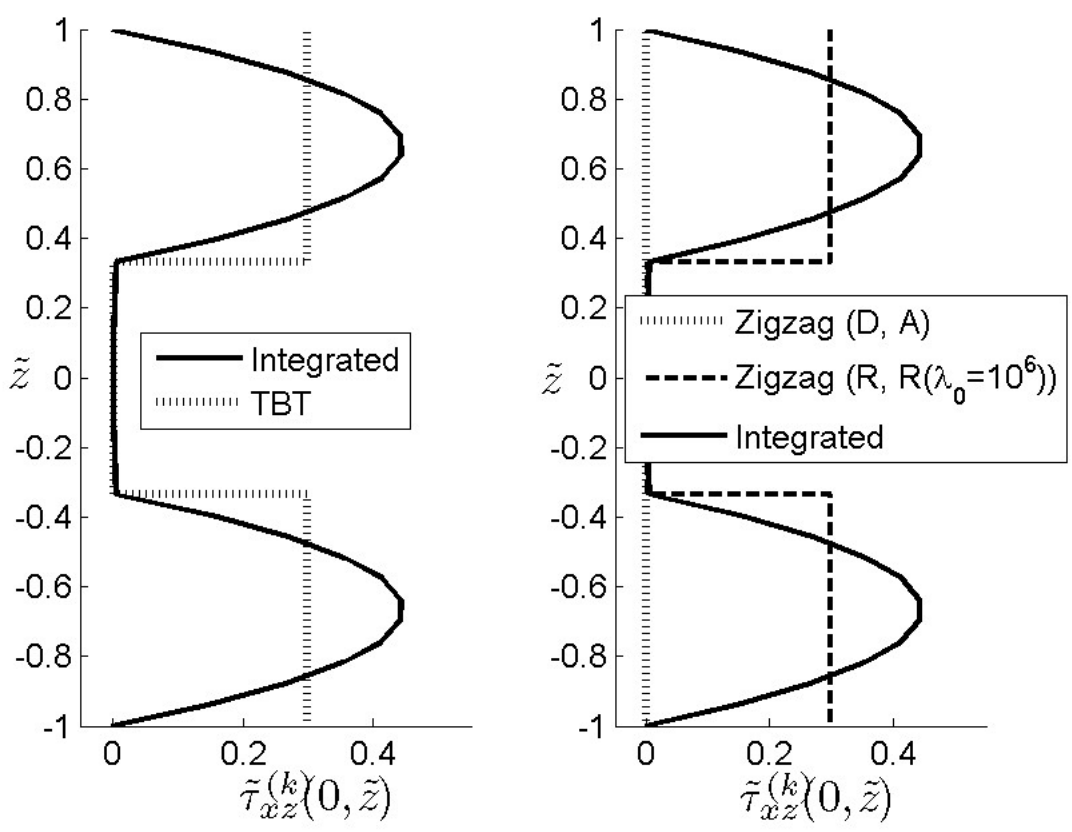

Figure 22. Cantilevered beam, laminate B: Normalized transverse shear stress at clamped end. 

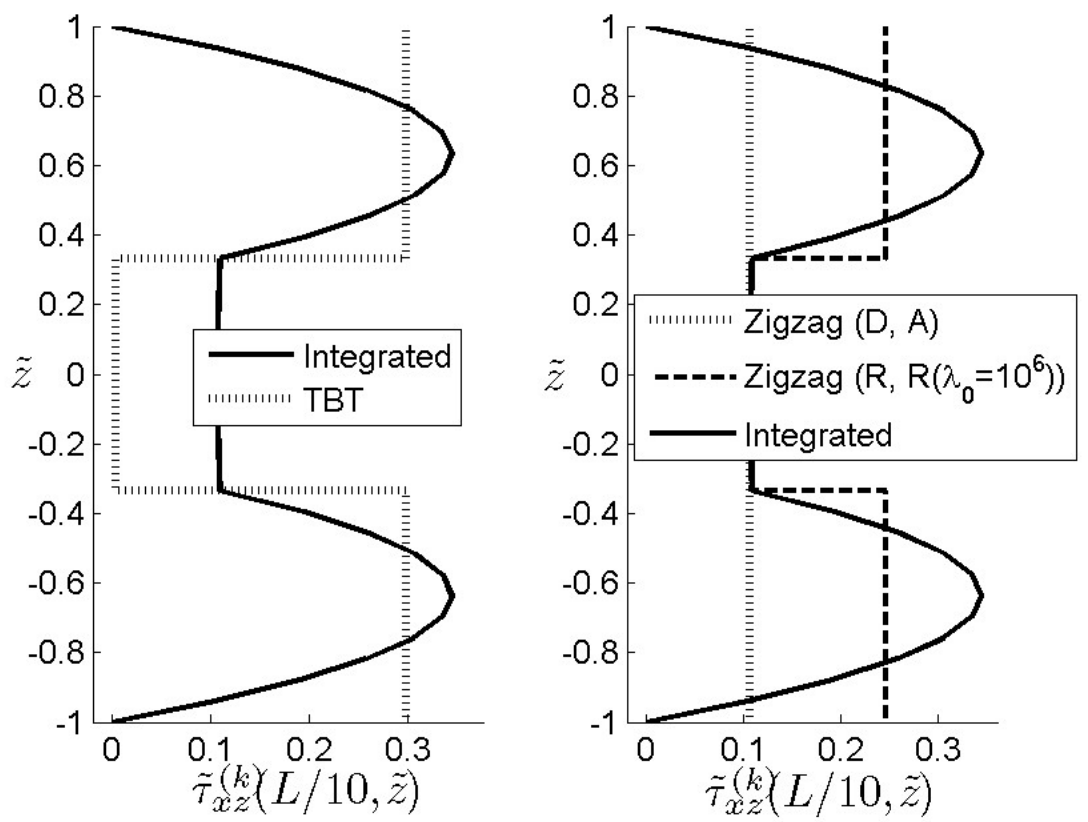

Figure 23. Cantilevered beam, laminate B: Normalized transverse shear stress at $\mathrm{x}=\mathrm{L} / 10$.
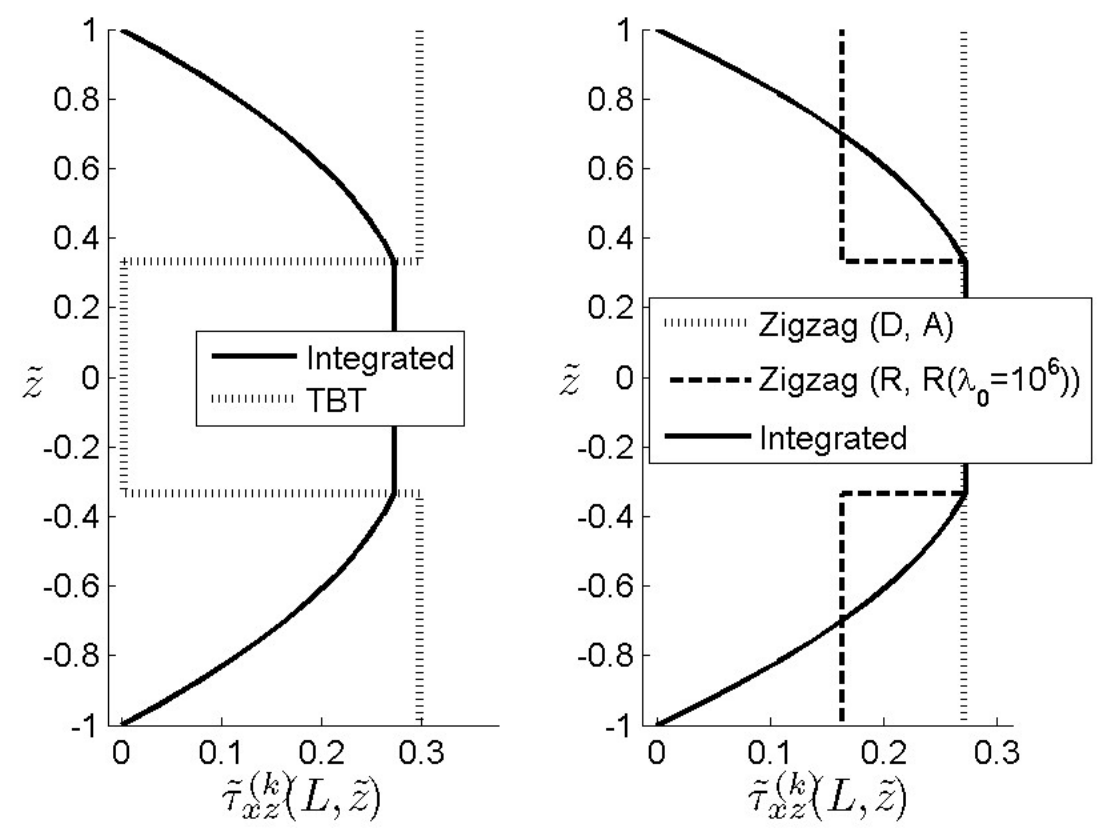

Figure 24. Cantilevered beam, laminate B: Normalized transverse shear stress at free end. 
Laminate B has three equal-thickness layers, a symmetric - with respect to the mid-plane transverse anisotropy, and a soft inner $(k=2)$ layer, such that $G_{x z}^{(1)} / G_{x z}^{(2)}=100$. For this laminate, Timoshenko solution is overly stiff, with the maximum deflection underestimated by about $50 \%$ (Figure 21). The refined zigzag theory results are consistently accurate. In contrast to Timoshenkotheory solution, the present zigzag theory models a changing - with respect to the axial location transverse shear stress distribution across the cross-sections (Figures 22-24). Closely correlated with the results pertaining to laminate A (Figure 20), a correct shear force distribution is obtained with the present theory, once again contrasting with Di Sciuva's theory in which $\tilde{V}_{x}$ vanishes at the clamped end $^{17}$.

\section{CONCLUSIONS}

A new refined zigzag theory for composite and sandwich beams was presented. The theory is consistently derived from the virtual work principle, and employs a novel form of the zigzag function that does not enforce continuity of the transverse shear stress across the beam cross-sections. The theory was assessed by way of exact solutions derived for simply supported and clamped thick beams made of highly heterogeneous material lay-ups. A Timoshenko-type shear correction factor is not used within the present theory. Results obtained with other beam theories, exact elasticity, and finite element solutions were used to assess the predictive capability of the present theory. The refined theory was shown to model accurately the overall beam stiffness as well as the displacement, stress, and stress resultant quantities. Importantly, the theory is devoid of the major shortcomings of the previous zigzag models, i.e., the shear force inconsistency and the related clamped boundary condition difficulties. Finally, the present theory may be readily extended to comparable plate and shell theories from which useful $C^{0}$-continuous finite elements may be developed. 


\section{ACKNOWLEDGMENTS}

The first author would like to acknowledge the support for this work provided by the Floyd L. Thompson NASA Fellowship. The third author gratefully acknowledges NIA - National Institute of Aerospace (Hampton, VA) for inviting and hosting him as a Visiting Researcher in the framework of “2007 Visitor Program” and acknowledges Politecnico di Torino for supporting his research activity at NIA in the framework of "Young Researchers Program" (2006 and 2007).

\section{REFERENCES}

1. Timoshenko S., (1921), On the correction for shear of differential equations for transverse vibrations of prismatic bars, Philos. Mag. Series, 41: 744-746.

2. Liu D. and Li X., (1996), An overall view of laminate theories based on displacement hypothesis, Journal of Composite Materials, 30(14): 1539-1561.

3. Ambartsumian S. A., (1964), Theory of anisotropic shells, NASA TTF-118.

4. Sun C. T. and Whitney J. M., (1973), Theories for the dynamic response of laminated plates, AIAA Journal, 11(2): 178-183.

5. Di Sciuva M., (1987), An improved shear-deformation theory for moderately thick multilayered anisotropic shells and plates, Journal of Applied Mechanics, 54: 589-596.

6. Di Sciuva M., (1984), A refinement of the transverse shear deformation theory for multilayered orthotropic plates, Proceedings of 7th AIDAA National Congress, October 1983, also published on L'aerotecnica missili e spazio, 62: 84-92.

7. Di Sciuva M., (1984), A refined transverse shear deformation theory for multilayered anisotropic plates, Atti Accademia delle Scienze di Torino, 118: 279-295.

8. Di Sciuva M., (1986), Bending, vibration and buckling of simply supported thick multilayered orthotropic plates: an evaluation of a new displacement model, Journal of Sound and Vibration, 105: $425-442$. 
9. Murakami H., (1986), Laminated composite plate theory with improved in-plane responses, Journal of Applied Mechanics, 53: 661-666.

10. Di Sciuva M., (1985), Development of an anisotropic, multilayered, shear-deformable rectangular plate element, Computers and Structures, 21(4): 789-796.

11. Di Sciuva M., (1985), Evaluation of some multilayered, shear-deformable plate elements, Proceedings of the $26^{\text {th }}$ Structures, Structural Dynamics and Materials Conference, AIAA/ASME/ASCE/AHS-Paper 85-0717: 394-400.

12. Di Sciuva M., (1990), Further refinement in the transverse shear deformation theory for multilayered composite plates, Atti Accademia delle Scienze di Torino, 124(5-6): 248-268.

13. Di Sciuva M., (1992), Multilayered anisotropic plate models with continuous interlaminar stresses, Composite Structures, 22(3): 149-168,

14. Averill R. C., (1994), Static and dynamic response of moderately thick laminated beams with damage, Composites Engineering, 4(4): 381-395.

15. Averill R. C. and Yuen Cheong Yip, (2006), Development of simple, robust finite elements based on refined theories for thick laminated beams, Computers \& Structures, 59(3): 529-546.

16. Umasree P. and Bhaskar K., (2006), Analytical solutions for flexure of clamped rectangular cross-ply plates using an accurate zig-zag type higher-order theory, Composite Structures, 74: 426-439.

17. Tessler A., Di Sciuva M. and Gherlone M., (2007), Refinement of Timoshenko beam theory for composite and sandwich beams using zigzag kinematics, NASA-TP-2007-215086, National Aeronautics and Space Administration, Washington, D.C.

18. Tessler A., Di Sciuva M. and Gherlone M., (2007), A refined linear zigzag theory for composite beams: reformulation of zigzag function and shear stress constraints, VI International Symposium on Advanced Composites and Applications for the New Millennium, Corfù, Greece, 16-18 May 2007

19. Mindlin R. D., (1951), Influence of rotatory inertia and shear deformation on flexural motions of isotropic elastic plates, ASME Journal of Applied Mechanics, 18: 31-38. 
20. Reissner E., (1945), The effect of transverse shear deformation on the bending of elastic plates, ASME Journal of Applied Mechanics, 12: 69-77.

21. Pagano N. J., (1969), Exact solutions for composite laminates in cylindrical bending, Journal of Composite Materials, 3: 398-411.

22. Di Sciuva M., Gherlone M. and Librescu L., (2002), Implications of damaged interfaces and of other non-classical effects on the load carrying capacity of multilayered composite shallow shells, International Journal of Non-Linear Mechanics, 37, 851-867.

23. Zienkiewicz O. C. and Taylor, R. L., (1989), The finite element method, Fourth Edition, McGraw-Hill Book Company, London

24. MSC/MD-NASTRAN, Reference Guide, Version 2006.0, MSC Software Corporation, Santa Ana, CA. 
Table 1. Mechanical material properties.

\begin{tabular}{|c|c|c|}
\hline $\begin{array}{l}\text { Material } \\
\text { type }\end{array}$ & $\begin{array}{l}E_{x}^{(k)}[\mathrm{GPa}] \\
\text { (Young's } \\
\text { modulus) }\end{array}$ & $\begin{array}{l}G_{x z}^{(k)}[\mathrm{GPa}] \\
\text { (Shear } \\
\text { modulus) }\end{array}$ \\
\hline $\mathrm{a}$ & 73.0 & 29.2 \\
\hline $\mathrm{b}$ & 21.9 & 8.76 \\
\hline C & 3.65 & 1.46 \\
\hline $\mathrm{d}$ & 0.73 & 0.292 \\
\hline e & 0.219 & 0.088 \\
\hline $\mathrm{f}$ & 0.073 & 0.029 \\
\hline
\end{tabular}

Table 2. Stacking sequences of three-layered laminates

(layer sequence is in the positive $\mathrm{z}$ direction).

\begin{tabular}{|c|c|c|}
\hline Laminate & Thicknesses [cm] & Materials \\
\hline A & $(0.20 / 1.60 / 0.20)$ & $(\mathrm{a} / \mathrm{f} / \mathrm{b})$ \\
\hline B & $(0.66 / 0.66 / 0.66)$ & $(\mathrm{b} / \mathrm{e} / \mathrm{b})$ \\
\hline C & $(0.66 / 0.66 / 0.66)$ & $(\mathrm{d} / \mathrm{a} / \mathrm{c})$ \\
\hline
\end{tabular}

